\title{
Problems and Progress in Microswimming
}

J. Koiller, ${ }^{1}$ K. Ehlers, ${ }^{2}$ and R. Montgomery ${ }^{2}$

1 Laboratório Nacional de Computacão Científica, R. Lauro Muller 455, Rio de Janeiro, 22290160 , RJ, Brazil

2 Mathematics Department, University of California at Santa Cruz, Applied Sciences Building, Santa Cruz, CA 95064, USA

Received January 5, 1996; revised manuscript accepted for publication June 1, 1996

Communicated by Jerrold Marsden and Stephen Wiggins*

Dedicated to the memory of Juan C. Simo, a pioneer in the use of geometry to produce better analytical and numerical methods in mechanics

Summary. Stokesian swimming is a geometric exercise, a collective game. In Part I, we review Shapere and Wilczek's gauge-theoretical approach for a single organism. We estimate the speeds of organisms moving by propagating small amplitude waves, and we make a conjecture regarding a new inequality for the Stokes' curvature. In Part II, we extend the gauge theory to collective motions. We advocate the influx of nonlinear control theory and subriemannian geometry. Computationally, parallel algorithms are natural, each microorganism representing a separate processor. In the final section, open questions motivated by biology are presented.

Key words. Stokes' flows, geometric phases, nonholonomic control

MSC classification codes (1991): 76D07, 76Z10, 93B29, 93C10, 51P05, 53C05

PACS classification codes (1990): 47.15.Gf, 87.45.-k, 87.10.+e, 02.40.+m, 03.40.Gc

\section{Part I: Bachelor's Life at Low Reynolds Number}

... Microwildernesses exist in a handful of soil or aqueous silt collected almost anywhere in the world. They are at least close to a pristine state and still unvisited. Bacteria, protists, nematodes, mites, and other minute creatures swarm around us, an animate matrix that binds Earths surface. They are objects of potentially endless study and admiration, if we are willing to sweep our vision down from the world

\footnotetext{
* This paper was solicited by the editors to be part of a volume dedicated to the memory of Juan C. Simo.
} 
lined by the horizon to include the world an arm's length away. A lifetime can be spent in a Magellanic voyage around the trunk of a single tree. If I could do it all over again, and relive my vision in the twenty-first century, I would be a microbial ecologist. Ten billion bacteria live in a gram of ordinary soil, a mere pinch held between thumb and forefinger. They represent thousands of species, almost none of which are known to science.

-E. O. Wilson, from the end of his book, The Naturalist

\section{Introduction}

The motions of living beings inspired Leonardo da Vinci's engineering endeavors. Giovanni Borelli's De motum animalium (1680) was an early interdisciplinary effort. Two centuries later, biologists, importing ideas from Industrial Revolution scientists [C. Bernard (Les phénomenes de la vie, 1878) and L. Frederick (who coined the term "regulatory agencies" in 1885)], showed that control theory applies as well to living machines as for steam engines. More recently, N. Wiener pointed out many areas of contact between applied mathematics and biology. He introduced the word "cybernetics" in 1961.

The earliest life forms appeared 3600 million years ago. The first invertebrates appeared 1600 million years ago. Genus homo appeared about 1 million years ago and civilization arose about .01 million years ago. Bacteria and protozoa, among the first life forms, have had plenty of time to optimize their performance. ${ }^{l}$ Paradoxically, very few roboticists or control theorists have looked into microorganism motion and survival strategies. (An exception is R. S. Fearing [33].) We suggest a control-theoretic approach to microorganism motility. These ancient organisms may have used "modern" ideas from nonholonomic motion planning and parallel computing. But a disclaimer is in order. Biofluiddynamics has a long tradition and we are novices, so we ask for indulgence.

We became interested in the problems of microswimmers through the work of A. Shapere and F. Wilczek [75]. In 1989 two of us heard these two talk on their "gaugetheory" explanation for microorganism motion. We felt that their approach paved the way for other ideas from geometric mechanics [1]. We were working on geometric phases, constrained variational problems, and nonholonomic systems. In the Workshop on Geometric Mechanics, Rio de Janeiro, 1993, one of us (JK) outlined a "vakonomic" [3] mechanics approach for microorganism motion. (Juan Simo raised his eyebrows in his ironic but very supportive way.) In the winter quarter of 1993-1994, we organized

\footnotetext{
I John Cohen, Interdisciplinary talkfest prompts flurry of questions, Science 270 (24 Nov 1995), page 1294:

"Bring together 100 of the best and brightest young (under age 45) scientists for 3 days to listen to talks from the cutting edge of a variety of disciplines, and what do you get? An endless barrage of questions, as participants try to slake their intellectual thirst in fields far removed from their own.... In a discussion at the end of the talks, the audience began peppering the engineers with questions and ideas about applying the microlessons of biological systems. Why not model a miniature motor after a flagella, exquisite micromotors that propel cells around the body? How about linking silicon devices to cells?"
} 
a seminar on microorganism motion at Santa Cruz. With the help of S. Wiggins and R. Murray we went down to Caltech, where we met Professors T. Y. Wu and C. Brennen (who not only did seminal work in the seventies [84], [19], [26], but have encouraged and influenced a great deal of other people's research). K. Ehlers, who was doing his thesis with R. Montgomery, found Professor Berg's beautiful book on bacterial motion. Together with the Brazilian biophysicists studying magnetotactic bacteria (Darci Esquivel, Marcos Farina, Henrique Lins de Barros), we had the privilege of having Prof. Berg visiting Rio in May 1995. Ehlers and Montgomery came down to participate. The list of problems in the last section came from these discussions.

The Stokes' approximation-neglecting the inertial terms in the Navier-Stokes equations-is valid for microorganisms (see, e.g., [81]). ${ }^{2}$ Their lives are dominated by two properties of their aqueous environment. These are viscous drag and Brownian motion. Due to drag, inertia plays essentially no role for the microswimmer and this is why the Stokes' equation is used instead of the Navier-Stokes. ${ }^{3}$ To illustrate this, H. Berg calculated in his book [4] that an $E$. coli coasts a distance small compared to the diameter of a hydrogen atom when it "throws in the clutch" and stops actively swimming. However, E. coli can never actually stop moving due to Brownian motion of the surrounding liquid. It is knocked around on the average of one body-width per second. $E$. coli cannot chase nutrients but relies on diffusion to take the nutrients to it. See the subsection on feeding in the problem list at the end. These two basic properties and various of their consequences are beautifully discussed in Purcell's article "Life at Low Reynold's Number" [71] which we urge all of our readers to read.

It is fashionable nowadays to mention possible applications: ${ }^{4}$

(i) "Peristaltic" (= low Reynolds) pumps. Two of us (RM and KE) met in July 1994 with engineers at the actuators and sensors division of EECS in Berkeley to investigate the possibilities of building a peristaltic pump based on Kurt's ideas. These engineers were in the process of building something very close. Instead of both walls of the pipe vibrating, they had only one wall. There was some disagreement about the underlying physics. We felt that the bulk of the transport might be accounted for by Stokes' equation. They used "acoustic streaming" which mathematically amounts to including part of the nonlinear inertial term of Navier-Stokes as a perturbation. In any case, their tiny pump can be built on a computer chip and could eventually be used for cooling or actuating.

(ii) Oil recovery. Suppose one can construct small, mobile robots, which move about in underground porous media and are able to "sniff" hydrocarbon molecules and follow their gradients. Why not imitate E. coli's use of chemotaxis ("sniffing" [4]) to locate oil patches? In the same vein, an exploration of Mars by a swarm of mini-robots is being planned [34].

(iii) Health. Unfortunately, there are not many studies relating motility to infectivity. But recent work may start to reverse this situation: strategies for vaccine development

\footnotetext{
${ }^{2}$ This fact seems to have been first noticed by Wilhelm Ludwig [57], whose work in the thirties remained forgotten for a long time. (G. Taylor, in his seminal 1951 work [79] did not refer to it.)

${ }^{3}$ It is worth comparing with high Reynolds swimming and flying; see [24], [54], [55].

${ }^{4}$ It is useful to know how to swim at low Reynolds numbers. Prof. H. Berg told us that he has a Boston Globe clipping saying that several unfortunate people were drowned when a large tank of molasses broke apart in 1912.
} 
are being attempted that rely on changing the genetic plans for the flagellum. Pathogenic E. coli, a major cause of infant mortality in third world countries, is the microorganism of choice. ${ }^{5}$

This paper is organized as follows. In Section 2 we review the gauge theory for one microswimmer; swimming ellipses and other examples from K. Ehlers' thesis are outlined. A result on motions induced by small amplitude waves is presented in Section 3: we add a bit of flesh to a general theorem in microswimming first brought to our attention by A. Shapere's thesis [76]. This is the "tangent plane approximation" for estimating the net swimming velocity. In Section 4 we outline our main new idea for collective swimming. It has been observed that there can be an advantage with cooperative microswimmers' behavior. In principle, this can be analyzed by extending Shapere and Wilczek's gauge-theoretic framework and borrowing ideas from nonholonomic control theory. In Section 5 we work out some "toy" examples to illustrate this. In Section 6 we give an annotated list of mathematical problems in microswimming. ${ }^{6}$ Most of them are real biological problems that came out of discussions with $\mathrm{H}$. Berg (misunderstandings are our responsibility!).

\section{The Geometric Description of a Single Swimming Microorganism}

No more pleasant sight has ever yet come before my eye than these many thousands of living creatures, seen all alive in a little drop of water, moving among one another, each creature having its own proper motion.

A gauge theory for the motion of an isolated, free-swimming microorganism $\mathcal{R}$ was proposed by Shapere and Wilczek in 1989 [75]. They considered the "envelope" model for ciliates, but with appropriate rewording, the same ideas hold for flagellar propulsion and more realistic models of ciliary motion [16], [17], [18], [84]. In this section we summarize the main features. For details, see also K. Ehlers' thesis [28].

One distinguishes from the outset abstract (or intrinsic) shapes $s$ and instantaneous intrinsic shape changes $\dot{s}$, from located shapes $q: s^{2} \rightarrow \mathcal{B} \subset \mathbb{R}^{3}, \mathcal{B}=\partial \mathcal{R}$, and corresponding boundary velocities $\vec{v}$. The set of located shapes forms our configuration space $\mathcal{Q}$, and the set of abstract shapes, denoted $\mathcal{S}$, is the quotient of $\mathcal{Q}$ by the group $G$ of rigid motions. Motion of the body in the fluid is an indirect result of the intrinsic shape changes. A more familiar example is a cat dropped upside down, which is able to reorient itself doing clever shape maneuvers (the physical constraint of zero angular momentum is satisfied throughout [62]).

A two-step procedure is commonly used by fluid mechanists. Fix a particular configuration of an isolated organism, and first consider a trial boundary condition $\vec{V}$ along the located boundary of the body, corresponding to the intrinsic infinitesimal shape deformation.

\footnotetext{
${ }^{5}$ For instance, scientists have been able to use bacteria to introduce genes into animal cells. See [77].

${ }^{6}$ We did some leisure reading too; some interesting sources are [27], [42], [23], [74], [67].
} 
There is an unavoidable ambiguity: $\vec{V}$ plus an infinitesimal rigid motion $X$ (consisting of infinitesimal translation and rotation) is as good as $\vec{V}$ in the sense that intrinsic shape deformation is the same. The correct $\vec{V}$ is picked out of the collection of $\vec{V}=\vec{V}_{\text {trial }}+X$ by adding the physical restriction that $\vec{V}$ induces no net force or torque on the fluid.

\subsection{Definitions and Notations}

The propulsion operator of a single microorganism is the linear map $\mathcal{L}$ that associates to a trial vectorfield $\vec{V}$ along the boundary the corresponding total force and torque $(\vec{F}, \vec{T})$ acting on the organism. ${ }^{7}$ Restricted to infinitesimal rigid motions, it is called the resistance operator $\mathcal{G}$ [40], which is represented by a $6 \times 6$ symmetric, positive definite matrix. Properties of $\mathcal{G}$ are summarized in Section 5.

In order to compute $\mathcal{L}$ one needs the first-order germ of the solution of Stokes' equations with boundary condition $\vec{V}$. If the whole solution $\hat{\vec{V}}$ is known, then $\mathcal{L}$ can also be computed from the asymptotics of $\hat{\vec{V}}$ at infinity (see [75], [28]).

The operator

$$
A=\mathcal{G}^{-1} \mathcal{L}
$$

is called the Stokes' connection 1-form. Its range is the Lie-algebra $s E(3)$. For a given intrinsic shape deformation $\dot{s}_{s}$ and located boundary q, there is a unique vectorfield $\vec{v}$ along the located boundary satisfying $A \cdot \vec{v}=0$ and consistent with $\dot{s}$. It gives the correct infinitesimal motion of the body in the fluid corresponding to the intrinsic shape deformation $\dot{s}$. The passage from the trial solution to the correct solution may be written (with some abuse of notation) as

$$
\vec{v}=(I-A) \vec{V}
$$

\subsection{Fluid Dynamics Background}

The fluid dynamics of swimming microorganisms are dominated by shear stresses. Inertia plays no role-any motion of the microorganism is communicated to the entire fluid without delay. The swimmer is not subjected to a net force or torque.

The equations of motion, known as Stokes' equations, are

$$
\begin{aligned}
\partial_{j} \sigma_{i j} & =0, \\
\partial_{i} v_{i} & =0,
\end{aligned}
$$

where

$$
\sigma_{i j}=-p \delta_{i j}+\mu\left(\frac{\partial v_{i}}{\partial x_{j}}+\frac{\partial v_{j}}{\partial x_{i}}\right) .
$$

Here $v_{i}$ is the fluid velocity and $p$ the pressure. The boundary conditions are no slip:

$$
\vec{v}=\vec{V} \text { on the boundary, }
$$

\footnotetext{
${ }^{7}$ This name was proposed by Purcell.
} 
where $\vec{V}=\vec{V}(x)$ is a given vectorfield on the boundary representing the organism (e.g., the "trial" deformation of the previous section), together with

$$
\vec{V}=0 \text { at infinity. }
$$

The stress tensor, $\sigma_{i j}$, represents the $i$ th component of the force per unit area exerted across a plane with normal in the $j$ direction. The total force exerted on the fluid is then given by the formula $F_{i}=-\int \sigma_{i j} \hat{n}_{j} d A$, where the integral can be taken over any surface enclosing the organism (since the divergence of $\sigma$ is zero). In short,

$$
\vec{F}=-\int \sigma \cdot \hat{n} d A
$$

Analogously, the total torque (with respect to an arbitrary origin 0 ) is given by

$$
\vec{T}=-\int \vec{r}_{0} \times(\sigma \cdot \hat{n}) d A
$$

If we put these together, we get the propulsion operator

$$
\mathcal{L}(V)=(\vec{F}, \vec{T})
$$

The resistance operator $\mathcal{G}$ is simply the propulsion operator restricted to infinitesimal isometries. Such an isometry is represented by a vectorfield $\vec{x} \rightarrow \vec{U}+\vec{\omega} \times \vec{x}$ where $\vec{U}$ represents the constant velocity and $\vec{\omega}$ represents the constant angular velocity. Let $V_{\vec{U}, \bar{\omega}}$ be the restriction of this vectorfield to the boundary of the organism. Then

$$
\mathcal{G}(\vec{U}, \vec{\omega})=\mathcal{L}\left(V_{\vec{U}, \vec{\omega}}\right)
$$

Remark. For those familiar with the mechanical connection associated to the $N$-body problem, note the similarity. $\mathcal{L}$ plays the role of the total angular momentum and $\mathcal{G}$ plays the role of the locked inertia tensor.

\subsection{Geometric Description of a Swimming Stroke}

Consider a moving cell membrane. We model it by a one-parameter family $q(t)$ of embeddings of the sphere $S^{2}$ into $\mathbb{R}^{3}$. The set of all embeddings of the two-sphere into three-space forms an infinite-dimensional manifold denoted by $\mathcal{Q}$. It is the underlying configuration space for swimming, and $q(t)$ is a curve in it. The derivative $\dot{q}$ of $q(t)$ at $t=0$ is then a tangent vector to $\mathcal{Q}$ at the particular located shape $q(0)$. Write $\mathcal{B}$ for the image of the sphere under $q(0)$, that is, for the physical cell membrane at time 0 . Then $\vec{V}=\dot{q}$ is a map $\mathcal{B} \rightarrow \mathbb{R}^{3}$ which represents the infinitesimal deformations of the particles of the cell membrane. The set of all tangent vectors $\vec{V}$ will be denoted by $T \mathcal{Q}$ and forms the tangent bundle to our space of located shapes. They are the possible boundary values for Stokes' equations. (See immediately following equation (4) above.)

If the organism is not allowed to move freely while attempting some infinitesimal deformation $\vec{V}$-for example, if we pin it to the microscope slide by some means-then of necessity it will be subjected to a net force and torque (provided by the pins). These 
forces and torques are linear functionals of the boundary data $\vec{V}$. Now if we remove the pins and allow the organism to move freely in response to the same infinitesimal deformation $\vec{V}$, then it will rotate and translate infinitesimally by some amount $\vec{X}$. The physics that determine this response $\vec{X}$ are that the organism exerts no net force and torque on the fluid. In this manner we obtain an linear assignment $\vec{V} \mapsto \vec{X}=\vec{A}_{s}(\vec{V})$ from $T_{q} \mathcal{Q}$ to $\mathbb{R}^{6}$, the Lie algebra of infinitesimal Euclidean motions. This map $A$ is called the Stokes' connection and is summarized by equation (1).

Now let $\mathcal{S}$ denote the collection of all possible intrinsic shapes which the organism can assume. It is the quotient of $\mathcal{Q}$ by the group $G$ of rigid motions. Its tangent bundle $T \mathcal{S}$ is then the collection of all shapes, together with the infinitesimal deformations. A tangent vector can be thought of as a vectorfield $\vec{V}: \mathcal{B} \rightarrow \mathbb{R}^{3}$ modulo infinitesimal motions $\vec{X}$. A swimming stroke is represented by a closed loop in $\mathcal{S}$ :

$$
s:[0, T] \rightarrow \mathcal{S},
$$

with $s(0)=s(T)$. When the organism performs this stroke in the fluid, it will rotate and translate so as to never violate the no net force and torque law. Although the initial and final shapes are the same, with any luck, the initial and final positions are not: the organism has swum! The initial and final located shape will differ by some Euclidean motion $g(T) \in G$. Our first goal is to understand $g(T)$ as a function of the swimming stroke.

In order to find it, choose some arbitrary "lift" or realization of the unlocated swimming stroke $s(t)$ by some curve $q(t)$ in $\mathcal{Q}$. This curve is not the physically accurate motion; rather, the correct located shape of the organism can be described by $g(t) \cdot q(t)$. The group elements $g(t) \in G$ provide the necessary corrections to our rather arbitrary "gauge" $q(t)$. With a bit of work (see Shapere and Wilczek [75]), one can show that $g$ satisfies the first-order ordinary linear time-dependent differential equation:

$$
\frac{d g}{d t}=g(t) \cdot A_{q(t)}(\dot{q}(t))
$$

(Caveat: a minus sign may appear depending on if you define the force and torque exerted by the fluid on the body or vice versa.)

Because the group of Euclidean motions is not Abelian, the solution is not as straightforward as one would hope. Physicists call the solution a "path-ordered exponential":

$$
\begin{aligned}
g(T) & =\bar{P} \exp \int_{0<t<T} A(t) d t \\
& =I+\int_{0<t_{1}<T} A\left(t_{1}\right) d t_{1}+\int_{0<t_{1}<t_{2}<T} A\left(t_{1}\right) A\left(t_{2}\right) d t_{1} d t_{2}+\cdots
\end{aligned}
$$

For large deformations, $g(T)$ must, in general, be found numerically. ${ }^{8}$ The following recent references may be useful for those interested in pursuing numerical work (we apologize for omissions): [83], [35], [31], [69], [72], [73].

\footnotetext{
${ }^{8}$ The authors have little computational background. Juan Simo's work combined deep abstract thinking together with tremendous numerical ability. We think Juan would enjoy this problem.
} 
Let us summarize what we have said so far, introducing the corresponding geometric language as we go. A swimming stroke is a closed loop in the space $\mathcal{S}$ of abstract shapes. The resulting distance (and reorientation) suffered as a result this stroke is the holonomy of this loop with respect to the Stokes' connection. The physical motion $q(t)$ representing where the cell membrane is in space at any instant $t$ of time is the horizontal lift of the loop in $\mathcal{S}$ to a (nonclosed) curve in $\mathcal{Q}$ with respect to the Stokes' connection. Either process of lifting or finding the holonomy involves solving the linear differential equation (8), which is also known as the equation of parallel transport.

\subsection{The Curvature Approximation Formula}

For small swimming motions $g$ can be estimated (to second order in the amplitude of the deformation) using the curvature of the connection form $A$.

Let $\left\{v_{n}\right\}$ be a basis for the vectorfields on the surface of a given located shape $q$. Define $\mathcal{F}_{m n}$ to be the infinitesimal Euclidean motion given by the coupling of the modes $v_{m}$ and $v_{n}$ (e.g., Fourier modes). The $\mathcal{F}_{m n}$ are nothing more than the components of the curvature two-form $\mathcal{F}$ of the connection form $A$, evaluated at the shape $q$ contracted with the vectors $v_{m}$ and $v_{n}$. A formula for $\mathcal{F}_{m n}$ is

$$
\mathcal{F}_{m n}=A\left(\left[v_{n}^{h}, v_{m}^{h}\right]\right),
$$

where

$$
\left[v_{n}^{h}, v_{m}^{h}\right]=\left(v_{n}^{h} \cdot \nabla\right) \hat{v}_{m_{\text {|shape }}^{h}}^{h}-\left(v_{m}^{h} \cdot \nabla\right) \hat{v}_{n_{\text {|:hape }}^{h}}
$$

is the Lie bracket. The hat indicates the Stokes' extension of the boundary condition to the fluid; the fluid response in a neighborhood of the boundary is necessary in order to compute the derivatives. The superscript $h$ denotes "horizontal projection"-which in practice means subtracting $A_{q}(v)$ from the input boundary conditions so that their Stokes' extensions lead to no net force or torque on the fluid.

Once we have the components of the curvature calculated at a particular shape we can approximate the connection form in a neighborhood of that shape. Let $a_{m}$ be the coordinates associated to the $v_{m}$. A boundary condition can then be written $v=\sum a_{m} v_{m}$, and the $a_{m}$ are to be thought of as the amplitudes. Then,

$$
\begin{aligned}
\mathcal{F}_{\mid s} & =\sum_{m<n} \mathcal{F}_{m n} d a_{m} \wedge d a_{n} \\
& =\sum_{m<n} d\left(a_{m} \mathcal{F}_{m n} d a_{n}\right) .
\end{aligned}
$$

Hence $A \cong \sum a_{m} \mathcal{F}_{m n} d a_{n}+$ exact. So if a swimming motion is gauge-parameterized by

$$
s(t)=q+\sum a_{n}(t) v_{n},
$$

where $q$ is a given located shape, then substituting the approximation for $A$ into formula (9), we obtain an approximation for the net motion associated to the periodic swimming stroke:

$$
\bar{P} \exp \int_{0}^{l} A(t) d t=I+\int \sum_{m<n} \mathcal{F}_{m n} a_{m} \dot{a}_{n} d t+O\left(|a|^{3}\right) .
$$




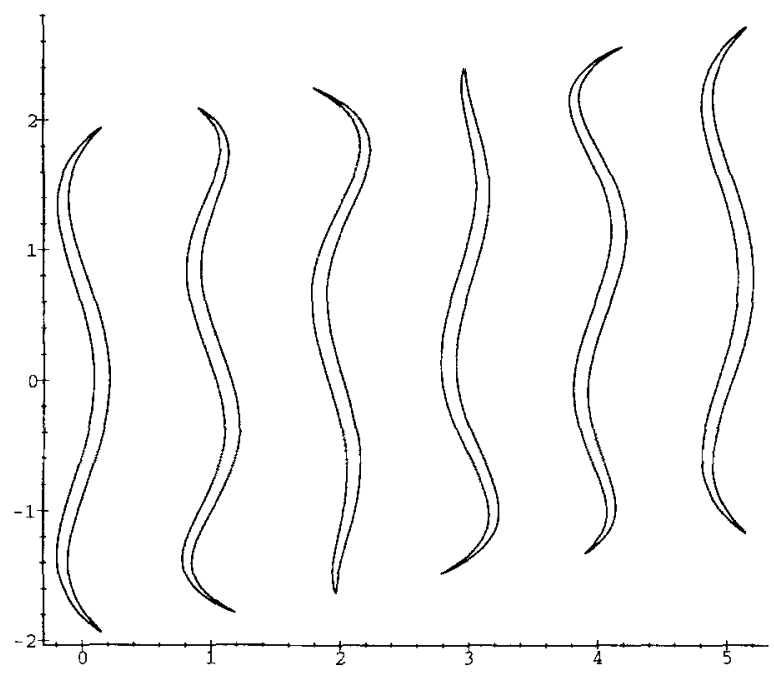

Fig. 1. Tubatrix model at times $t=0,0.2,0.4,0.6,0.8$, and 1.0 .

So, to describe arbitrary small amplitude swimming motions of a base shape $s$, we must compute the curvature at a given shape. The resulting matrix encodes the essential dynamics of the problem. To compute the curvature we need the following ingredients:

1. a fixed basis for the vectorfields defined on the exterior of the base shape,

2. solutions to Stokes' equations with boundary conditions specified on the base shape,

3. an expression for the Lie bracket in the appropriate coordinates,

4. an efficient way to compute the net force and torque associated to an arbitrary vectorfield specified on the base shape (the operator $\mathcal{L}$ ),

5. the vectorfields defined on the exterior of the base shape corresponding to rigid rotations and rigid translations.

\subsection{Examples}

For two-dimensional problems, complex variable techniques for the biharmonic equation [66], [30] are available. Following the steps above makes the calculations quite algorithmical. One of us (KE) computed swimming strokes for deforming ellipses [28]. In his thesis the curvature for an ellipsoid of revolution and for the axial modes on a cylinder were also investigated. The cylinder problem can be done analytically. Components of the ellipsoid curvature are calculated using Maple ${ }^{(\mathcal{C}}$. The curvatures for a number of other examples are described in the next section.

A swimming pattern for a model Tubatrix aceti is depicted in Figure 1. To represent this long, thin organism that swims by passing undulatory waves down its body like an eel, one takes a large eccentricity ellipse and deforms it with purely imaginary coefficients. The predicted translation per stroke is $18 \%$ of its length. A real Tubatrix is slightly slower; it has been observed to translate approximately $16.5 \%$ of its length per stroke. 
The interested reader may see animation of various swimming models using the software Maple ${ }^{\mathcal{C}}$. Contact the authors by e-mail for the code (kehlers@bert.ucsc.edu, rmont@cats.ucsc.edu, or jair@ server02.Incc.br).

\section{Traveling Waves on Surfaces and Curvature Asymptotics}

Imagine a series of waves of very small amplitude $a$ and very short wavelength $\lambda$ traveling continuously along a sphere or other surface with wave speed $c$. With what speed $V$ will the surface translate through the fluid? In this section we will derive the estimate:

$$
V \sim \frac{1}{2} c\left(\frac{a}{\lambda}\right)^{2}
$$

by using the curvature approximation formula. Then we will relate it to the "tangent plane approximation" of Shapere [76].

This estimate for $V$ can be guessed at on dimensional grounds, once we realize that the curvature approximation formula implies that $V$ must be proportional to $a^{2}$. It is hidden in formulas of Blake and others for special types of waves on special types of surfaces. (See Brennen [18] for plane waves on planes and Blake for Legendre waves on spheres [16]. These and other examples are worked below.) But we have not found this simple form written down explicitly anywhere. We learned of it from Berg, who in turn learned of it through a recent conversation with Blake. It is a starting point in trying to understand whether it is possible for the mystery swimmer (see section 6.2 below) to swim using this imagined strategy.

The careful reader will have a number of serious questions regarding this whole discussion. What is a "traveling wave on a surface," especially some general asymmetric surface? What is its wave length? Is it a local or global object? Et cetera. We will only touch these questions below. But first we proceed as follows. Represent the surface wave by the vector function $\vec{w}(x, t)$ where $x$ is a material point on the surface boundary and $x+\vec{w}(x, t)$ represents that point at time $t$. Take some $L_{2}$ orthonormal basis $\left\{\vec{u}_{m}\right\}$ for the space of vector functions $\mathcal{T}$ along the surface and expand $w$ in terms of them:

$$
\vec{w}(x, t)=\Sigma a_{m}(t) \vec{u}_{m}(x)
$$

with the condition that

$$
\int_{0}^{T} a_{m}(t) d t=0 .
$$

Also expand the Stokes' curvature in this basis:

$$
\mathcal{F}=\Sigma_{m<n} \mathcal{F}_{m n} d a_{m} \wedge d a_{n}
$$

Remember that $\vec{w}$ and hence the $a_{m}$ are meant to be very small. Then the curvature approximation formula states that to one period $T$ of the wave motion the cell membrane has translated by approximately $\int_{0}^{T} \Sigma_{m<n} \mathcal{F}_{m n} a_{m}(t) \frac{d}{d t} a_{n}(t) d t$. (Hereafter, we only con- 
sider the translational part of the curvature.) Hence the mean velocity $\vec{V}$ of its motion is

$$
\vec{V}=\frac{1}{T} \int_{0}^{T} \Sigma_{m<n} \mathcal{F}_{m n} a_{m}(t) \frac{d}{d t} a_{n}(t) d t .
$$

Imagine a wave where only two modes are coupled:

$$
\vec{w}(x, t)=a\left\{\cos (\omega t) \vec{u}_{m}(x)+\sin (\omega t) \vec{u}_{n}(x)\right\} .
$$

We calculate $\Sigma_{m<n} \mathcal{F}_{m n} a_{m} \frac{d}{d t} a_{n}=a^{2} \omega \mathcal{F}_{m n} \cos ^{2}(\omega t)$. Since the average of $\cos ^{2}$ is $1 / 2$, we arrive at the velocity formula:

$$
\vec{V}=\frac{1}{2} a^{2} \omega \mathcal{F}_{m n}
$$

On the other hand, the wave speed $c$ of a true plane wave is related to its wavelength and frequency by $c=\omega \lambda$. Then our original estimate for $V$ becomes $V \sim \frac{1}{2} a^{2} \omega \frac{1}{\lambda}$. Comparing formulae, we obtain

$$
\mathcal{F}_{m n} \sim \frac{1}{\lambda}
$$

relating the curvature components to the effective wavelength $\lambda$ of the waves in our basis. But which $\lambda$ do we use, the one for $m$ or the one for $n$ ? And again, what do we even mean by the wave number for waves on a surface?

Do we have a guess for the leading term? If we scale the whole surface by an amount $\ell, \mathcal{F}_{m n}$ scales by $\ell^{-1}$. This suggests that the leading term is related to the diameter of the body. (The answer does depend on dimension; for plane swimming $\mathcal{F}_{m n}$ is invariant under rescaling.)

This is a good time to go to the examples.

\subsection{The Swimming Slab}

(See [24], especially equation (3.14), p. 26, [76, ch. 4], [18], and [28, final section].)

Plane waves of a fixed wavelength $2 \pi / k$ travel along an infinite sheet. The sheet is represented by the $x y$ plane and the half-space $z \leq 0$ represents the body of the "microorganism." The waves travel in the $x$ direction and we will suppose that they have no components in the $y$ direction. Then an appropriate basis is

$$
\begin{aligned}
& u_{m}^{+}=\cos (m k x) e_{1}, \\
& u_{m}^{-}=\sin (m k x) e_{1}, \\
& v_{m}^{+}=\cos (m k x) e_{3}, \\
& v_{m}^{-}=\sin (m k x) e_{3} .
\end{aligned}
$$

The curvature entries involving different modes $m$ and $n$ with $m \neq n$ are all zero. So the curvature comes in $4 \times 4$ blocks indexed by the mode $m$. Remember the curvature is a 
vector-valued matrix. In this case its components are all proportional to $e_{1}$, the direction of wave propagation, so we will drop the vector index and think of the curvature as scalar-valued. Relative to the basis above this matrix is $m k$ times a matrix whose entries consist solely of $1 \mathrm{~s}, 0 \mathrm{~s}$, and $-1 \mathrm{~s}$.

Take a traveling wave:

$$
\vec{w}(x, t)=a \cos (k x-\omega t) e_{3}
$$

representing a vertical deformation. Expanding the cosine we find that $w=a\left\{\cos (\omega t) v_{1}^{+}\right.$ $\left.(x)+\sin (\omega t) v_{1}^{-}(x)\right\}$, precisely the above form. Using the above formula for the velocity we find that it is

$$
V=\frac{1}{2} a^{2} \omega k
$$

This is a special case of formula (3.14) of Childress's book [24, p. 26].

\subsection{Circles and Cylinders}

(See [15], [75], and [28].)

The surface is represented by the interior of a circle or cylinder. In the case of the cylinder we only allow deformations that are independent of the axial $(z)$ coordinate and that have no component in this direction. Then the cylinder problem reduces to that of the circle. Identify vectors in the plane with complex numbers. Then an appropriate basis for the deformations is

$$
u_{m}(\theta)=e^{i m \theta}
$$

and

$$
v_{m}(\theta)=i e^{i m \theta} .
$$

The only nonzero curvature components are of the form $\mathcal{F}\left(u_{m}, v_{n}\right)$ with $m-n= \pm 1$, and they are $\pm i 2 m$. Again $\mathcal{F}_{m n} \sim \frac{1}{\lambda} \sim m$.

\subsection{The Sphere}

(See [16], [75], and [28].)

The surface is the unit sphere. The appropriate basis for surface deformations is the vector spherical harmonics, written $Y_{J, l, m}$. They form an $L_{2}$-orthonormal basis of eigenfunctions for the vector-valued Laplacian. The index $J$ represents the total angular momentum (as in quantum mechanics) and is asymptotic to the square root of the eigenvalue of the spherical Laplacian. The only modes that couple in the curvature tensor are those with the same $J$ and for which one of the indices $m$ and $l$ differ by 1 . The corresponding curvature components are asymptotic to a constant times $J$.

The simplest case is that of purely radial deformations which only depend on the latitudinal angle $\theta$. These can be described by Legendre polynomials $P_{n}$. When normalized this yields the basis

$$
\left\{\sqrt{\frac{4 \pi}{2 n+1}} P_{n}(\cos (\theta)) e_{r}\right\} .
$$


The only nonzero components are $\mathcal{F}_{n, n+1}$ and these are asymptotic to $\frac{-1}{4 \pi} n$ (cf. pp. $60-61$ of Shapere's thesis).

\subsection{The Ellipse}

One of us (Ehlers) found the curvature at an ellipse using complex variable techniques. Under the conformal mapping

$$
w(\xi)=R\left(\xi+\frac{\epsilon}{\xi}\right)
$$

the unit circle gets mapped to an ellipse with major and minor axes having lengths $2 R(1+\epsilon)$ and $2 R(1-\epsilon)$. We use the basis

$$
\begin{array}{lll}
u_{n}(w)=e^{i n \xi}, & w=w(\xi), & |\xi|=1, \\
v_{n}(w)=i e^{i n \xi}, & w=w(\xi), & |\xi|=1,
\end{array}
$$

for deformations of the ellipse. (Warning: we have shifted our index by 1 compared to [28].) It is convenient to combine the $u$ and $v$ in expressing the curvature using the fact that $v_{n}=i u_{n}$. For $\alpha, \beta$ complex numbers write

$$
\mathcal{F}\left(\alpha u_{m}, \beta u_{n}\right)=\alpha \beta \mathcal{F}_{m n}+\bar{\alpha} \beta \mathcal{F}_{\bar{m} n}+\alpha \bar{\beta} \mathcal{F}_{m \bar{n}}+\bar{\alpha} \bar{\beta} \mathcal{F}_{\bar{m} \bar{n}},
$$

thus defining the complex curvature components $\mathcal{F}_{m n}, \mathcal{F}_{\bar{m} n}$, etc. (Of course they are easily related to the usual $\mathcal{F}\left(u_{m}, u_{n}\right), \mathcal{F}\left(u_{m}, v_{n}\right)$, etc.) The result is that

$$
\mathcal{F}_{m \bar{n}}=m \epsilon^{l}, \quad m-n=2 l+1>0, \quad m, n>0 .
$$

The components with $m-n$ even and the $\mathcal{F}_{m n}, \mathcal{F}_{m n}$ are zero. $\mathcal{F}_{\bar{m} n}$ can be obtained from $\mathcal{F}_{m \bar{n}}$ by skew-symmetry. Note that again

$$
\mathcal{F}_{m n} \sim \min \{m, n\} .
$$

\subsection{Discussion and Problems}

In all these cases the nonzero entries of the curvature go as

$$
\left|\mathcal{F}_{m n}\right| \sim|m|
$$

for $|m|,|n| \gg 1$, provided the basis $\left\{\vec{u}_{m}\right\}$ is appropriately normalized and the absolute value $|m|$ is appropriately interpreted (for the sphere $|(J, l, m)|=|J|$ ). In the symmetric examples of the plane, circle, and sphere, the curvature matrix breaks up into small blocks, no more than $4 \times 4$. For the ellipses infinitely many modes enter, but still $\left|\mathcal{F}_{m n}\right| \leq$ $\min \{|m|,|n|\}$. This suggest that the wavelength $\lambda$ corresponds to the mode number $m$. In the symmetric examples above the basis we used was a normalized eigenbasis for the Laplacian on that surface. More precisely, each component of the vectorfield along the surface is an eigenfunction of the Laplacian. The mode numbers were proportional, for large $m$, to the square root of the corresponding eigenvalue $E(m)$ for the Laplace operator. 


\subsection{Spectral Methods}

In the examples above the eigenbasis for the surface deformations that we used were taken from the Laplacian operator. A more natural operator is the stress tensor $\sigma$ contracted with the normal vector $\vec{n}$ to the surface boundary. Fix a located boundary shape $\mathcal{B}$, which we think of as a surface in space representing the cell membrane. (See Section 2.3.) Recall (Section 2.3) that the tangent space at $\mathcal{B}$ to our configuration space $\mathcal{Q}$ of all located shapes is the linear space $T_{\mathcal{B}} \mathcal{Q}$ consisting of all vector-valued maps $\mathcal{B} \rightarrow \mathbb{R}^{\ni}$. The stress tensor as defined by equation (4) and the Stokes' extension $\vec{v}$ of the deformation $\vec{V}$ defines a linear operator $\mathcal{P}: \mathcal{T} \rightarrow \mathcal{T}$ by mapping $\vec{V}$ to

$$
\mathcal{P}(\vec{V})=: \sigma_{\mid \mathcal{B}} \cdot \vec{n}
$$

Here $\vec{n}$ is the unit normal vector to the surface $\mathcal{B}$. Physically, $\mathcal{P}(\vec{V})(x)$ is the force per unit area that the fluid exerts on the boundary at $x$, given that the boundary deforms according to $\vec{V}$.

The bilinear form

$$
(W, V)=\int_{\mathcal{B}} \vec{W} \cdot \mathcal{P} \vec{V} d^{2} S
$$

defines a Riemannian metric on our configuration space $\mathcal{Q}$. This remarkable fact will be discussed and proved in a forthcoming paper. The horizontal space, or physically allowable (zero force and torque) deformations, and the "vertical space" (span of the infinitesimal rigid motions $\vec{X}$ ) are orthogonal complements to each other relative to this metric. It follows that the Stokes" connection is a "mechanical" or "Riemannian submersion" induced connection, just like the connection for the falling cat.

A fundamental fact is that the operator $\mathcal{P}$ is symmetric and positive definite. (This result was published by $H$. A. Lorentz in 1906). See [40, Section 3.5] for a proof in a slightly more general situation. Positive definiteness is seen physically by observing that

$$
E(\vec{V})=\int \vec{V} \cdot \mathcal{P}(\vec{V})
$$

represents the mechanical power expenditure due to the deformation $\vec{V}$. The propulsion operator $\mathcal{L}$ (see Section 2.2), which is the basic ingredient of the Stokes' connection, can be written in the form $\mathcal{L}(\vec{V})=(\vec{F}(\vec{V}), \vec{T}(\vec{V}))$ where

$$
\vec{F}=-\int \mathcal{P}(\vec{V}) d S
$$

represents the total force and

$$
\vec{T}=-\int \vec{r}_{0} \times \mathcal{P}(\vec{V}) d S
$$

the total torque. See Section 2.2.

Some thought suggests that a "Poincaré inequality" of the form

$$
|\mathcal{F}(\vec{u}, \vec{v})| \leq\|\vec{u}\|\|\mathcal{P}(\vec{v})\|+\|\vec{v}\|\|\mathcal{P}(\vec{u})\|
$$


ought to be true. Here the norms of deformation vectorfields, $\vec{u}$, $\vec{v}$, are their $L_{2}$ norms defined by integration over the surface. Let $\left\{\vec{u}_{m}\right\}$ be an $L_{2}$ orthonormal basis for the space of vector functions on the surface, consisting of the eigenvectors of $\mathcal{P}$ :

$$
\mathcal{P}\left(\vec{u}_{m}\right)=\lambda_{m} \vec{u}_{m},
$$

with corresponding real positive eigenvalues $\lambda_{m}$. Such a "Poincaré" inequality would yield the curvature bounds

$$
\mathcal{F}_{m n} \leq \lambda_{m}+\lambda_{n}
$$

for the components of the curvature $\mathcal{F}$ relative to this basis.

Remark. This hypothetical curvature bound and the calculations of the above examples suggest that (at least for those examples) there will be a simple relationship between $\mathcal{P}$ and the "godfather of all operators," the Laplacian.

3.6.1. A Spectral Algorithm. We now present a theoretical algorithm for solving the Stokesian swimming based on the spectral analysis of $\mathcal{P}$. The crux of the Stokesian swimming problem is to find an effective means of computing (17) and (18) for every trial boundary condition on every located boundary shape $\mathcal{B}$. The spectral decomposition of $\mathcal{P}$ may provide such a means, and it is the heart of this algorithm. As far as we know, this proposed algorithm is new.

Step 1. Find $\left\{\vec{u}_{m}\right\}$, an $L_{2}$ orthonormal basis for the space of vector functions on the surface, consisting of the eigenvectors of $\mathcal{P}$. Also find the corresponding real positive eigenvalues $\lambda_{m}$.

Numerically, this step can be implemented via singularity collocation methods [39], [83]. Peskin's method [69] is another interesting possibiliy, since it yields $\vec{v}_{m}$, given $\mathcal{P}$.

Step 2. Define the "linear" and "angular momentum" vectors of a given trial boundary vectorfield $\vec{V}$

$$
\begin{gathered}
\vec{L}(\vec{V})=\int \vec{V} d S, \\
\vec{M}(\vec{V})=\int \vec{r}_{0} \times \vec{V} d S,
\end{gathered}
$$

so that

$$
\begin{aligned}
& \vec{F}_{m}=\vec{F}\left(\overrightarrow{u_{m}}\right)=-\lambda_{m} \vec{L} \overrightarrow{u_{m}}, \\
& \vec{T}_{m}=\vec{T}\left(\overrightarrow{u_{m}}\right)=-\lambda_{m} \vec{M} \overrightarrow{u_{m}},
\end{aligned}
$$

are the associated forces and torques for our eigenbasis. See Section 2.2.

This illustrates that in some sense the eigenvectorfields are "principal directions" for Stokesian deformations. 
Step 3. Expand the given trial vectorfield $\vec{V}$ in terms of the eigenbasis:

$$
\vec{V}(x)=\Sigma a_{m} \vec{u}_{m}(x)
$$

Then, by linearity,

$$
\vec{F}(\vec{V})=\Sigma a_{m} \vec{F}_{m}, \quad \vec{T}(\vec{V})=\Sigma a_{m} \vec{T}_{m}
$$

Step 4. Compute the resistance matrix $\mathcal{G}$ of the shape $\mathcal{B}$. See Section 2.2. (Recall that this transformation is obtained by applying $\mathcal{L}$ to infinitesimal translations and rotations restricted to the boundary, so it can be computed, for example, by applying step 3 to these translation and rotation vectorfields as trials.)

Step 5. Invert $\mathcal{G}$ and then either apply it directly to $(\vec{F}, \vec{T})$ of step 3, to obtain $X=A(V)$, or apply it to the $\left(\vec{F}_{m}, \vec{T}_{m}\right)$ of step 2 to obtain the Lie-algebra elements

$$
X_{m}=\mathcal{G}^{-1}\left(\vec{F}_{m}, \quad \vec{T}_{m}\right)=-\lambda_{m} \mathcal{G}^{-1}\left(\vec{L}_{m}, \quad \vec{M}_{m}\right)
$$

and hence the complete connection one-form at our given shape: $A=\Sigma X_{m} d \vec{u}_{m}$. We have the linear relation

$$
X=\Sigma a_{m} X_{m}
$$

The Stokes' flow for $X$ is the "counterflow" associated to the trial $\vec{V}$ which gives the actual flow: $\vec{v}=\vec{V}-\hat{X}$. This ends the algorithm.

Remarks. The mechanical power expenditure of the trial vector (21) is

$$
E=\Sigma \lambda_{m} a_{m}^{2} \text {. }
$$

The power expenditure of the corrected vectorfield is

$$
\begin{aligned}
E & =\langle\mathcal{P}(\vec{v}), \vec{v}\rangle \\
& =\langle\mathcal{P}(\vec{V}), \vec{V}\rangle-\vec{F} \cdot \vec{U}+\vec{T} \cdot \vec{\omega},
\end{aligned}
$$

where $\vec{v}=\vec{V}-X$ is horizontal, $X=(\vec{U}, \vec{\omega})$ is the rigid motion producing the counterflow, and $\mathcal{L}(\vec{V})=(\vec{F}, \vec{T})$. Equation (23) seems to indicate that higher modes are more effective for swimming. However, (24) shows they consume more power.

The algorithm may be appended to give a possible alternative to calculating the curvature.

In a future publication we plan to present examples where the spectral decomposition of $\mathcal{P}$ can be obtained analytically. For the sphere of radius $a$ we have found its spectrum to be given by (here $n=-2,-3, \ldots ; \chi_{n}, \varphi_{n}, p_{n}$ are solid spheric harmonics of order $n$ )

Family 1: $\lambda_{n}^{(1)}=\mu(n-1) / a, v_{n}=\nabla \times\left(\chi_{n} \vec{r}\right)$;

Family $2: \lambda_{n}^{(2)}=2 \mu(n-1) / a, v_{n}^{(2)}=\nabla \varphi_{n}$;

Family $3: \lambda_{n}^{(3)}=\frac{2 \mu}{a}(2 n+4+3 / n)$.

The eigenvector is

$$
v_{n}^{(3)}=\frac{r^{2}-a^{2}}{2 \mu(2 n+1)} \nabla p_{n}+\frac{n r^{2 n+3}}{(n+1)(2 n+1)(2 n+3)} \nabla\left(\frac{p_{n}}{r^{2 n+1}}\right)
$$




\subsection{The Tangent Plane Approximation: An Open Problem}

This is taken from Shapere's thesis. Again, imagine a very small-amplitude, shortwavelength deformation of the surface. Follow the following "algorithm":

- Step 1. Near each point $P$ replace the surface by its tangent plane at $P$ and the wave by the corresponding periodic deformation of the plane.

- Step 2. Calculate the flow field and resultant velocity at infinity, $u(P)$ due to this planar deformation.

- Step 3. Replace the original boundary conditions at the surface with the new boundary condition $u(P)$. Solve Stokes' equations. Find the resulting flow at infinity, thus obtaining the net translation by finding the resulting force.

The rationale in this construction is that the wave is so small that infinity for the planewave approximation (steps 1 and 2) is right near the actual original surface. The first step is the problematic one. What do we mean by "the corresponding periodic deformation" of the tangent plane? This only appears to make sense asymptotically. Consider a family

$$
\vec{w}(x, t ; a, \lambda)=a \vec{W}(x, t ; \lambda)
$$

with $a$ being the amplitude and $\lambda$ some measure of wavelength as above. Express $\vec{W}$ relative to Gaussian normal coordinates $x$ centered at an arbitrary point $x_{0}$ of the surface. Rescale the coordinate $x$ to focus attention on the point of contact. Thus consider $\vec{W}\left(x_{0}+\lambda x, t ; \lambda\right)$. Now hope (or assume) that in the limit the corresponding function of $x$ converges on compact sets to a plane wave or at least to a periodic wave on the tangent plane. The phase, frequency, and amplitude of this wave will vary as $x_{0}$ varies.

Perhaps this can be made more rigorous or palatable by multiplying $\vec{W}$ or its rescaled version by cut-off functions and then analyzing the Fourier transforms as $\lambda \rightarrow 0$. Some help here might be buried somewhere in the book Geometric Asymptotics [38] or in the larger pseudodifferential calculus literature.

\section{Part II: Social Life at Low Reynolds Number}

On the basis of organization we may regard the [protozoan] cell as a huge computer on a molecular scale, with a very broad and complicated program.

\section{-T. L. Jahn and E. C. Bovee}

At some unrecorded point in time, perhaps very early in the history of protozoa, certain of the one-celled animals began to form societies of cells-communities in which different cells had different functions - and from these societies the metazoa, the many-celled animals, are believed to have evolved.

- Helena Curtis

Protozoa are 'simple' only in that they are generally tiny and have few observable body structures. Physiologically and behaviorally, they are not simple, carrying out all functions carried out by multicellular forms within a restricted framework. They have mechanoreceptors, chemoreceptors, and photoreceptors ... At the time 
of conjugation in ciliates, or fusion of gametes in flagellates, two individuals come together, and this might suggest some form of mutual or individual attraction.... For certain peritrichous ciliates, the microconjugant, which is free-swimming, can identify the macroconjugant, which is sessile, by chemicals given off by the latter. When a microconjugant passes within one millimeter of a macroconjugant (a considerable distance in its size scale), it stops and undertakes a search until it finds the other.

- Hubert and Mable Frings

\section{Nonholonomic Path Planning Inside Stokes' Flows}

It may be a good idea to begin with an example.

\subsection{Can Scallops Swim at Zero Reynolds Number?}

A scallop is any Stokesian free swimmer whose shape can be parameterized by a line segment (one-dimensional). The "scallop theorem" states that scallops are non-swimmers. If the shape is changed and returned to the original by the reverse path (reparameterizations not only allowed but encouraged) the initial and end states coincide. A formal statement can be found in Childress [24].

A basic fact stressed ad nauseum is that the shape space of a successful Stokesian swimmer must be at least two-dimensional. A swimming sequence of a two-hinged organism is illustrated in [71]. We now know ${ }^{10}$ why it swims from left to right (a challenge in Purcell's paper).

It turns out that, after all, scallops may swim if there are two or more of them, and if they cooperate. This is one of the main points of this second half of our paper. The reader is challenged to provide a choreography. Scallops $a$ (Alice) and $b$ (Bob) are ready to perform their pas-de-deux in Figure 2.

\subsection{General Framework}

Let $N$ organisms $\mathcal{R}_{j}$ with deformable boundaries $\mathcal{B}_{j}$ swim at zero Reynolds number. ${ }^{\text {II }}$ The grand configuration space $\mathcal{Q}=\mathcal{Q}_{1} \times \cdots \times \mathcal{Q}_{N}$ consists of $N$-tuples of maps $q_{j}: S^{2} \rightarrow \mathcal{B}_{j}$ from the sphere to the Euclidean space, describing the located shape of each body. More precisely, the map gives the location of every material particle on the boundary. We mark a special point on every organism (a "nose").

4.2.1. A Fiber Bundle Festival. The Euclidean group $G=S E(3)$ acts on the right on the $q_{j}$ by composition and hence defines a diagonal action on $\mathcal{Q}$. The quotient space $\mathcal{S}$ is

\footnotetext{
${ }^{9}$ The name is due to Purcell [71].

10 We thank Rubens Sampaio Filho.

11 Microorganisms may divide or conjugate, so strictly speaking $N$ may vary in time.
} 


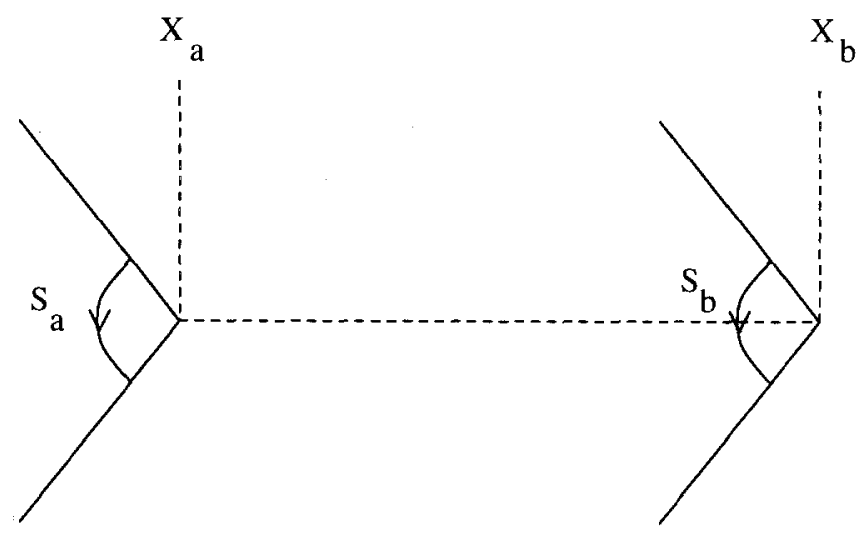

Fig. 2. Scallops Alice and Bob swimming happily.

called the overall shape space. We take the diagonal action because the relative positions and orientations of the bodies matter (otherwise there would be no collective swimming effects). Let $\mathcal{S}_{j}=\mathcal{Q}_{j} / S E(3)$ be the individual shape spaces. Notice that $S$ is a principal bundle over $S_{1} \times \cdots \times S_{N}$ with fiber $S E(3)^{N-1}$.

(i) Suppose that there is a collective "intelligence" that simultaneously controls all intrinsic shape changes, $\dot{s}=\left(\dot{s}_{1}, \ldots, \dot{s}_{N}\right)$. Since we marked a nose on each body, we have a fiber bundle $\mathcal{Q} \rightarrow \mathcal{S}_{1} \times \cdots \times \mathcal{S}_{N}$. It is a principal bundle whose fiber is the direct product $S E(3) \times \cdots \times S E(3)$ (N-copies). Each $S E(3)$ acts on its own organism alone. We will see that collective swimming is not described by a principal bundle connection; rather, it is given by an Ehresman connection. The distribution has codimension $6 \mathrm{~N}$ as expected, but it is not $(S E(3))^{N}$ equivariant. It is equivariant only under the diagonal $S E(3)$ action.

(ii) Another point of view is more "individualistic." Each body $j$ controls only its own boundary shape deformation $\dot{s}_{j}$. Ignoring the presence of other bodies, we define $N$ principal bundles $\pi_{j}: \mathcal{Q}_{j} \rightarrow \mathcal{S}_{j}$ with connection forms as in Part 1 . The total space $\mathcal{Q}$ is the product of the $\mathcal{Q}_{j}$.

From these individual connections we can reconstruct the global Ehresman connection, using the reflection method [40] to account for the mutual effect of the organisms. We next describe this connection from both points of view, (i) and (ii), and show that they are equal.

4.2.2. The Connection Form: Viewpoint (i). Associated to a set of trial boundary conditions, there is a unique Stokes' flow external to the union of the domains. The grand propulsion operator $\mathcal{L}_{\text {grand }}$ has now a range of $N$ forces and $N$ torques, whose calculation again requires only the first-order jet of the Stokes' flow computed along the boundaries. Restricted to $N$ (generally distinct) infinitesimal rigid motions as boundary conditions, one gets a $6 N \times 6 N$ grand-resistance matrix $\mathcal{G}_{\text {grand }}$ [40]. The connection form is $A_{\text {grand }}=\mathcal{G}_{\text {grand }}^{-1} \mathcal{L}_{\text {grand. }}$. The corrected boundary velocities are given by

$$
\left(\left.\vec{u}_{1}\right|_{B_{1}}, \ldots,\left.\vec{u}_{N}\right|_{B_{N}}\right)=\left(I-A_{\text {grand }}\right)\left(\left.\vec{U}_{1}\right|_{B_{1}}, \ldots,\left.\vec{U}_{N}\right|_{B_{N}}\right) .
$$


where the $\vec{U}_{j}$ are arbitrary trial vectorfields on the boundaries consistent with the intrinsic shape changes.

4.2.3. The Connection Form: Viewpoint (ii). We now present the connection form, viewpoint (ii). Although it will be equal to (i), its ingredients are useful for comparing the collective swimming against the individual efforts. With several organisms interacting, there are two additional complications: first, the counterflows $\hat{X}_{j}$ induce different translations and rotations on every body. Second, the "intrinsic" shape deformation performed by each isolated organism must be modified in order to yield a desired translation (due to the influence of flows generated by the others). To address these two issues, we follow the biological rationale that each organism is a separate processor, independently making its "decentralized decisions" (but taking into consideration the "social" environment).

We call $\left.\vec{v}_{j}\right|_{B_{j}}$ a virtual located shape deformation (of the $j$ th organism). Solving Stokes' equations for the whole space external to $\mathcal{R}_{j}$ (ignoring the other organisms, that is filling them in with fluid) produces a fluid flow that we also denote by $\vec{v}_{j}$. In the geometric language, this defines a connection on the $j$ th bundle $\mathcal{Q}_{j} \rightarrow \mathcal{S}_{j}$. We repeat: the idea behind this construction is the following: if all the other bodies $k \neq j$ could correctly "guess" the vectorfield $\vec{v}_{j}$ and choose to change shape accordingly, then the $j$ th organism would not be able to tell (hydrodynamically) that the others were present and could proceed as if they were not.

For microorganisms close enough to interact hydrodynamically, virtual shape deformations are "adjusted" to result in prescribed actual deformations $\left.\vec{u}_{k}\right|_{B_{k}}$. In fact, besides its own self-generated virtual motion, the $k$ th organism is under the influence of ("feels") the flows generated by all others. Hence its actual deformation is given by

$$
\left.\overrightarrow{u_{k}}\right|_{B_{k}}=\left.\vec{v}_{k}\right|_{B_{k}}+\left.\sum_{j \neq k} v_{j}\right|_{B_{k}}
$$

It is important to verify that the off-diagonal terms (i.e., the other organism influences) $j \neq k$ produce no net force and torque on the $k$ th organism. This follows directly from the fact that the stress tensor for $\vec{v}_{k}$ is divergence-free, on the exterior to $\mathcal{R}_{k}$, the region of organism $k$.

Uniqueness theorems guarantee that (i) and (ii) produce the same solution. In practice, the extra work needed to compute the "grand" operators is equivalent (or worse) to the functional inversion involved in the iterative process.

We anticipate at this point a biological question:

Problem. Protozoa have a sense of "touch," i.e., have mechanical pressure transducers. Can they "compute" the virtual deformations (the modified shape deformations) whose implementation will result in true deformations?

\subsection{Discussion}

Suppose organism 1 wants to translate (and rotate) in the direction $X_{1}\left(X_{1} \in \operatorname{se}(3)\right)$. Let $v_{1}$ be a shape deformation that would yield $X_{1}$, given that no other organisms are present. Repeat this process for organisms 2 through $n$, arriving at shape deformations $v_{1}, \ldots, v_{n}$. 
Due to interactions between the flows the organisms will not move in direction $X_{i}$. The fields $u_{i}$ of equation (25) represent the two conditions. They result instead in motions $Y_{i}$. (As the distance between organisms tends to infinity, the $Y_{i}$ tend to the $X_{i}$.) On the other hand, $v_{i}$ is what organism $i$ actually controls. So we are left with the following inversion problem: given that the Stokes' flow $u$, with $u_{\mid \mathcal{B}}=u_{i}$, leads to desired motions $Y_{i}$, find individual deformations $v_{i}$ which lead, through equation (25), to the flow $u$. This is a problem of functional inversion. In control theoretical jargon, the inverse operator is called a feedback control transformation. In low Reynolds hydrodynamics literature, reflection methods [40] implement the functional inversion by an iterative process. In computer science terminology, each organism acts as a separate processor, working in parallel; interaction is implemented via the off-diagonal terms, or an equivalent domaindecomposition technique.

It remains to be seen whether these metaphors can be biologically meaningful. We summarize them in the words of Andrew Klinger:

Not only mathematical terms but also problem solving methods are inevitably modeled on Nature. Cooperation may not require direct communication. Domain decomposition could indeed be (our description of) the process, with no need for direct links. Assuming that the time scale for the individual organism 'awareness' to be small compared to the time scale of the actual motion, the collectivity may quite efficiently achieve 'virtual communication' by an iterative process of solving a local problem and then comparing the immediate results with the goals.

\subsection{Collective Path Planning in Stokes' Flows}

"The study of nonholonomic motion planning is in its infancy." (S. Sastry, R. Murray)

Nature has a 4 billion year expertise on nonholonomic control theory.

-the authors

Nonholonomic motion planning is a recent area of nonlinear control theory stimulated by robotics [53]. Some applications are airport baggage-trailer parking, satellite attitude control, multifingered manipulators, and ultrasonic motors. Recall the key notions. Let $y$ denote $n$-dimensional states and $r m$-dimensional control variables ( $m=2$ typically). The states obey an ODE of the form

$$
\dot{y}=\sum_{j=1}^{m} r_{j} Y_{j}(y) .
$$

Suppose that for every $y$, the Lie algebra spanned by the vectorfields $Y_{j}$, under Lie bracketing, spans the tangent space at $y$. By Chow's theorem [25] the system is controllable, in the sense that any state can be reached. One needs more or fewer "zig-zags" to steer toward the goal, depending upon the Lie algebra structure. Recently, several theoretical results have been obtained to quantify this notion and to obtain normal forms useful for engineering practice [53]. Control problems on a principal G-bundle were studied by Montgomery [63], [64]. 
Our viewpoint is that equation (25) may also be interpreted as a nonlinear control system of type (26), with the individual shape deformations $r_{j}=\dot{s}_{j}$ as controls. Thus Nature has a 3.6 (plus) billion year expertise on nonholonomic control theory. The control vectorfields are lifted by the linear connection operator to induced vectorfields along the boundaries of all the bodies, implementing the state changes.

Problem. Do some microorganisms actually perform collective path planning strategies to achieve a desired location? Can optimality criteria be defined? Do they play differential games (predator vs. prey)?

We have here a more general form of control problem on principal bundles. To finish this section, it is appropriate to distill an abstract nonsense summary. The following is a setting for "collective control problems" (generalizing [64]).

Collective Connections and Variational Problems. Let $\left(G_{i} \hookrightarrow Q_{i} \rightarrow S_{i}, A_{i}\right), i=$ $1, \ldots, N$, principal bundles with connection forms $A_{i}$. For simplicity take all $G_{i}$ equal to a same group $G$. Let $Q=Q_{1} \times \cdots \times Q_{N}$ and $\pi_{j}$ the projection over $Q_{j}$. Denote by $\operatorname{hor}_{i}\left(\dot{s}_{i}^{\text {virt }}\right)$ the horizontal lift operator of the $i$ th connection. We assume that hor ${ }_{i}$ can be extended to $\operatorname{Hor}_{i}\left(\dot{s}_{i}^{\mathrm{virt}}\right) \in T_{q} Q$, equivariantly under the diagonal action of $\mathrm{G}$, in such a way that $A_{j}\left(\pi_{* j} \operatorname{Hor}_{i}(\bullet)\right)=0$. Call $\dot{s}_{i}^{\text {virt }}$ a virtual rate of shape change. Dynamics in the state space $Q$ is governed by the joint lift of $\dot{s}_{i}^{\text {virt }}$, i.e.,

$$
\dot{q}=\sum_{i=1}^{N} \operatorname{Hor}_{i}\left(\dot{s}_{i}^{\mathrm{int}}\right)
$$

The constraints $A_{i}\left(\pi_{* i} \dot{q}\right)=0, i=1, \ldots, N$, are satisfied, but there is a subtle point here: $\left.\pi_{i}\right|_{*}(\dot{q})$ is in general different from $\dot{s}_{i}^{\text {virt }}$. So we cannot event start lifting a virtual curve $s^{\text {virt }}(t)$, because the actual shapes would change differently! We circumvent this difficulty by banishing virtual curves and allowing only the symbol $\dot{s}$ virt_regarded as "quasicontrols." A control protocol is a curve $s(t)=\left(s_{1}(t), \ldots, s_{N}(t)\right)$. The $N$ equations $\dot{s}_{i}=:\left.\pi_{i}\right|_{*}(\dot{q})$ bring into stage the observability issue. We must assume that the linear mapping $\left(\dot{s}_{1}^{\text {virt }}, \ldots, \dot{s}_{N}^{\text {virt }}\right) \rightarrow\left(\dot{s}_{1}, \ldots, \dot{s}_{N}\right)$ is invertible. The inverse is called the "feedback transformation," relating the true shape rates to "quasi-controls." Control curve $s(t)$ is lifted to $q(t)$ via (27).

Now, suppose $Q$ has a $G$-invariant Riemannian metric $g$. Defining length of curves $\left(s_{1}(t), \ldots, s_{N}(t)\right)$ as $\int \sqrt{g(\dot{q}, \dot{q})} d t$ produces a sub-Riemannian metric on $Q$. It is still sub-Riemannian in $Q / G$ (with the diagonal action).

\section{Toy Examples}

The reasonable microbiologist adapts himself to the microbial world. The unreasonable one persists in trying to adapt the microbial world to himself. Therefore, all progress depends on the unreasonable microbe. 


\subsection{Can Scallops Swim at Zero Reynolds Number?}

We now sketch some calculations for our problem (Section 4.1). Suppose that scallop $a$ (Alice) is trying to swim along the $x$-axis. Let $s_{a}$ be a parameter describing Alice's shape, $x_{a}$ her position. Alice's velocity is of the form $v_{a}=\kappa_{a}\left(s_{a}\right) \dot{s}_{a}$. The far field generated by Alice is of a dipole, whose associated stokeslets have opposite strength $4 \pi \mu \varphi_{a}\left(s_{a}\right) \dot{s}_{a}$. Functions $\kappa_{a}$ and $\varphi_{a}$ encode Alice's hydrodynamic characteristics.

Suppose that another scallop $b$ (Bob) is around. The joint states of Alice and Bob are represented by $y=\left(s_{a}, s_{b}, x_{a}, x_{b}\right)$, and the control system is given by

$$
\dot{y}=\dot{s}_{a} Y_{a}(y)+\dot{s}_{b} Y_{b}(y)
$$

with

$$
\begin{aligned}
& Y_{a}=\left(1,0, \kappa_{a}\left(s_{a}\right), \frac{\varphi_{a}\left(s_{a}\right)}{\left(x_{b}-x_{a}\right)^{2}}\right), \\
& Y_{b}=\left(0,1, \frac{\varphi_{b}\left(s_{b}\right)}{\left(x_{b}-x_{a}\right)^{2}}, \kappa_{b}\left(s_{b}\right)\right) .
\end{aligned}
$$

Exercise. Compute the Lie brackets of $Y_{a}$ and $Y_{b}$ and check that the system is controllable.

We observe that a variational problem, leading to a sub-Riemannian geometry is defined by the mechanical power expenditure. This is of the form

$$
g=g_{a}\left(s_{a}, \ell\right) \dot{s}_{a}^{2}+g_{b}\left(s_{b}, \ell\right) \dot{s}_{b}^{2}, \quad \ell=\left|x_{b}-x_{a}\right| .
$$

\subsection{Virtual vs. True Velocities on Stokeslets Fields}

Consider two stokeslets "a" and "b", point particles subject to external forces $\vec{F}_{a}, \vec{F}_{b}$ and having virtual velocities $\vec{V}_{a}, \vec{V}_{b}$ respectively. This means that $\vec{F}_{a}=-\mu K_{a} \vec{V}_{a}$ would relate force and velocity at $a$, if $b$ were not present. Likewise, $\vec{F}_{b}=-\mu K_{b} \vec{V}_{b}$.

Take the origin at $a$ and the positive y-axis along the line-segment $\bar{a} b$ and let $\ell$ be the distance between the two stokeslets. Denote by $\vec{U}_{a}=\left(U_{a x}, U_{a y}\right), \vec{U}_{b}=\left(U_{b x}, U_{b y}\right)$ the true velocities. From the analytical expression of a stokeslet field one constructs the mobility matrix

$$
\mathbf{A}=\left(\begin{array}{cccc}
1 & 0 & \frac{K_{b}}{8 \pi \ell} & 0 \\
0 & 1 & 0 & \frac{K_{b}}{4 \pi \ell} \\
\frac{K_{a}}{8 \pi \ell} & 0 & 1 & 0 \\
0 & \frac{K_{a}}{4 \pi \ell} & 0 & 1
\end{array}\right)
$$

relating the forces to the true velocities, that is,

$$
\left(U_{a x}, U_{a y}, U_{b x}, U_{b y}\right)^{\dagger}=\mathbf{A}\left(\frac{F_{a x}}{-\mu K_{a}}, \frac{F_{a y}}{-\mu K_{a}}, \frac{F_{b x}}{-\mu K_{b}}, \frac{F_{b y}}{-\mu K_{b}}\right)^{\dagger}
$$


Inverting the mobility matrix one gets the resistance matrix, which gives $\left(\vec{F}_{a}, \vec{F}_{b}\right)$ in terms of the true velocities. Thus

$$
\frac{\left(F_{a x}, F_{a y}\right)}{\mu K_{a}}=\left(\frac{-U_{a x}+\frac{K_{b}}{8 \pi \ell} U_{b x}}{1-\frac{K_{a} K_{b}}{(8 \pi \ell)^{2}}}, \frac{-U_{a y}+\frac{K_{b}}{4 \pi \ell} U_{b y}}{1-\frac{K_{a} K_{b}}{(4 \pi \ell)^{2}}}\right)
$$

(just exchange indices for the force in $b$ ). This is precisely equation (6.2-15) in [40], obtained via the reflection method (a tricky calculation, where one sums geometric series).

Exercise. The reader is invited to extend (31) to a more general form (31) where the line connecting the two stokeslets makes an angle $\alpha$ with the $x$-axis.

5.2.1. The Hydrodynamical Binary. Suppose that " $a$ " and " $b$ " can interact, but there are no external forces. The condition $\vec{F}_{\text {total }}=\vec{F}_{a}+\vec{F}_{b}=0$ yields, after a simple algebra,

$$
U_{a x}=-p(\ell) U_{b x}, \quad U_{a y}=-q(\ell) U_{b y}
$$

which

$$
p=\frac{A+\frac{B}{\ell}}{1+\frac{B}{\ell}}, \quad q=\frac{A+\frac{2 B}{\ell}}{1+\frac{2 B}{\ell}},
$$

and where the parameters are given by $A=K_{a} / K_{b}, B=K_{b} / 8 \pi$.

In general, the segment $\overline{a b}$ makes an angle $\alpha$ with the $x$-axis. By equivariance, we may apply (33) if we rotate the velocity vectors by $\exp i\left(\frac{\pi}{2}-\alpha\right)$. We get a distribution of planes in configuration space $\mathbb{R}^{2} \times \mathbb{R}^{2}=\left\{\left(x_{a}, y_{a}, x_{b}, y_{b}\right)\right\}$ given by

$$
\begin{aligned}
& \left(\sin \alpha U_{a x}-\cos \alpha U_{a y}\right)=-p(\ell)\left(\sin \alpha U_{b x}-\cos \alpha U_{b y}\right), \\
& \left(\cos \alpha U_{a x}+\sin \alpha U_{a y}\right)=-q(\ell)\left(\cos \alpha U_{b x}+\sin \alpha U_{b y}\right),
\end{aligned}
$$

where $\ell \cos \alpha=x_{b}-x_{a}, \quad \ell \sin \alpha=y_{b}-y_{a}, \quad \ell^{2}=\left(x_{b}-x_{a}\right)^{2}+\left(y_{b}-y_{a}\right)^{2}$.

Exercise. If $K_{a} \neq K_{b}$, the distribution is nonintegrable and of full rank. The whole configuration space is accessible.

We remark that if $K_{a}=K_{b}$ then $A=1$ and the functions $F, G$ become constant, $p=q \equiv 1$. This implies that the distribution, in this case defined by $\mathbf{U}_{a}=-\mathbf{U}_{b}$, is obviously integrable. The midpoint between " $a$ " and " $b$ " remains fixed.

\subsection{The Hydrodynamical Connection for $N$ Rigid Bodies}

5.3.1. The Setting. Consider $N$ rigid bodies moving in a viscous fluid. We assume that they can interact, say, by hydrodynamically negligible "rods," but there are no external forces. The configuration space is $Q=S E(3)^{N}$ (minus collisions), acted diagonally upon by the group of rigid motions $S E$ (3), yielding a principal bundle whose base is the shape space $S=Q / S E(3)=S E(3)^{N-1}$. 
The principal bundle for the motion of $N$ isotropic point particles is just $\left(\mathbb{R}^{3}\right)^{N}$ acted upon by the group of rigid motions of the space. For $N \geq 3$ this action is generally free; however this is not so for $N=2$, because rotations along the axis determined by the two particles do not act. We can make the action free by assuming that the particles move on a plane. The configuration space is now four-dimensional, and the group, threedimensional. The quotient space is parameterized by the distances $\ell>0$ between the two particles. ${ }^{12}$

5.3.2. Basic Facts. An isolated rigid body, moving in a Stokes' flow, is characterized by 21 parameters (twelve for two symmetric $3 \times 3$ matrices $K, \Omega$ and nine for a general matrix $C$ ). More generally, the collective motion of $N$ rigid bodies is characterized by a $6 N \times 6 N$ symmetric matrix [40, chapter 8$]$

$$
\mathcal{G}=\left(\begin{array}{ll}
\mathbf{K} & \mathbf{C}^{\dagger} \\
\mathbf{C} & \Omega
\end{array}\right)
$$

called the grand resistance matrix. $\mathbf{K}$ is formed by $N^{2} 3 \times 3$ blocks $K_{i j}$ (likewise $\mathbf{C}$, $\Omega$ ). Therefore

$$
K_{i j}^{\dagger}=K_{j i}, \quad \Omega_{i j}^{\dagger}=\Omega_{j i}
$$

$\mathcal{G}$ depends upon (i) the shapes and sizes of the particles, and (ii) the distances and orientations with respect to each other. $\mathcal{G}$ relates linearly the forces and torques acting on each and every one particle of the system with their velocities and spins with respect to the fluid at rest at infinity:

$$
\left(\vec{F}_{1}, \ldots, \vec{F}_{N}, \vec{T}_{1}, \ldots, \vec{T}_{N}\right)^{\dagger}=\mathcal{G}\left(\vec{U}_{1}, \ldots, \vec{U}_{N}, \vec{\omega}_{1}, \ldots, \vec{\omega}_{N}\right)^{\dagger}
$$

If $\mathrm{G}$ is known, we can obtain the total force $\vec{F}$ and torque $\vec{T}$ :

$$
\begin{aligned}
& \vec{F}=\sum_{i} \vec{F}_{i}=\sum_{j}\left(\sum_{i} K_{i j}\right) \vec{U}_{j}+\sum_{j}\left(\sum_{i} C_{i j}\right) \vec{\omega}_{j}, \\
& \vec{T}=\sum_{i} \vec{T}_{i}=\sum_{j}\left(\sum_{i} C_{i j}^{\dagger}\right) \vec{U}_{j}+\sum_{j}\left(\sum_{i} \Omega_{i j}\right) \vec{\omega}_{j} .
\end{aligned}
$$

5.3.3. An Isoholonomic Problem. The grand-resistance matrix has been mainly used for sedimentation studies, where the applied force is gravity. Here we suggest another application:

Robotics model. The bodies are articulated by "invisible rods" (i.e., hydrodynamically negligible) which can control the shape of the configuration.

\footnotetext{
${ }^{12}$ If you have your feet trapped on tar, follow this advice: make $N=3$ or 4 by putting your hands in too. You should be able to escape!
} 
Since actual motions inside the fluid satisfy the conditions of zero total force and total torque on the system, these conditions define a connection in the bundle. We call it the hydrodynamical connection of a system of rigid bodies.

The power, or instantaneous mechanical energy dissipation rate $E$ (i.e., the sum of the instantaneous rates at which the stresses acting over the surfaces of the bodies are doing work upon it) is given by

$$
\begin{aligned}
E & =-\sum_{i} \int_{S_{i}} d \vec{n}_{i} \cdot \sigma \cdot \mathbf{v}_{i} \\
& =-\sum_{i}\left(\vec{U}_{i}, \vec{\omega}_{i}\right) \cdot\left(\vec{F}_{i}, \vec{T}_{i}\right)^{\dagger} \\
& =\mu(\mathbf{U}, \omega) \mathcal{G}(\mathbf{U}, \omega)^{\dagger} .
\end{aligned}
$$

We get an isoholonomic variational problem [63] on configuration space $Q$ with Lagrangian $L=E$ given by (39) subject to the constraints $\vec{F}=0$ and $\vec{T}=0$.

5.3.4. Propositions. The following assertions must be true (a verification is in order):

(i) The local holonomy algebra of the hydrodynamical connection is all of se(3).

Digression. Guichardet explicitly identified what some call the "natural mechanical connection" associated to $N$ mass points moving in empty space [37]. The configuration space is $Q=\left(\mathbb{R}^{3}\right)^{N} . G=S E(3)$ acts on $Q$. Here the physical constraints are zero linear and zero angular momentum. The first leads to the conservation of center of mass, which means that for the Guichardet's connection no holonomy takes place in the translation subgroup. The usual reduced Guichardet connection is a connection on the $S O(3)$ principal bundle whose total space is the subspace $V$ of $\left(\mathbb{R}^{3}\right)^{N}$ of configurations whose center of mass is at the origin. (Remove collinear configurations to obtain a true principal bundle.) Guichardet's connection is defined on the reduced configuration space and its local holonomy algebra is all of $s o(3)$. This fact is well known to cats, athletes, and astronauts, which are able to perform interesting rotational maneuvers. Note: For molecular chemistry and robotics it should be important to extend Guichardet's connection to the case where instead of point particles one considers spinning rigid bodies, in which case the configuration space becomes $Q=S E(3)^{N}$.

Returning to the viscous fluid, if the bodies are so far apart that their interaction can be neglected, then $G$ becomes sparse, corresponding (after a trivial rearrangement) to $N 6 \times 6$ blocks of individual resistance matrices. More precisely, this six-diagonal form corresponds to writing sequentially the forces and torques of each particle on one side of the equation and the velocities and spins on the other. In other words, we assert that:

(ii) The hydrodynamical connection is asymptotically flat.

(iii) As a consequence, in the isoholonomic variational problem, in order for a system of bodies to translate, it may be an advantage to first cluster together to interact. 
5.3.5. Specific Examples. Happel-Brenner's treatise [40] is a treasure chest for those interested in concrete examples. For instance:

(i) Motions of N point particles on a line. Smoluchowski's formulas in [40, section 8.3] are a starting point to compute the hydrodynamical connection.

(ii) Two concentric spheres. The Stokes' flow is given in [40, section 7.8]. A configuration of the system is $\left(R_{1}, R_{2}\right) \in S O(3) \times S O(3)$, a moving frame for each sphere. Suppose one can control the relative attitude $S=R_{1} R_{2}^{-1}$. Given $S^{-1} \dot{S}$, what are the instantaneous angular velocities of the spheres, viewed externally?

(iii) Towing with stokeslets. Interpret the forces $\vec{F}_{a}, \vec{F}_{b}$ in equation (31)' as the controls, and positions $\left(x_{a}, y_{a}, x_{b}, y_{b}\right)$ as states. For $N$ point particles the controls are forces $\vec{F}_{j}, j=1, \ldots, N$, some of which can be inactive. Think of marble balls inside a syrup tank, some of which have iron cores and may be controlled by magnetic fields. Is the system of marbles controllable? Can just one iron ball control the whole system?

Remark. Because of the zero force and torque condition on free-swimming organisms, pure stokeslets have been considered "outlaws." Nevertheless we believe we have found a problem for example (iii). ${ }^{13}$ See [59] ("Insects set sail": cover of Nature, September $28,1995)$. In the next section we advertise other real biological problems-we believe the mathematical treatment can be very interesting.

\section{A List of Problems}

E. coli is an optimist.

-H. Berg

Some of these problems were collected during the winter 1993-1994 when we had a seminar in Santa Cruz, and some during the discussions we had in May 1995, during Howard Berg's visit to Rio. The first two problems are the ones of most interest to Berg and are probably of the greatest biological import.

\subsection{Feeding at Low Reynolds Number}

In trying to understand the mechanisms and strategies behind the myriad of feeding methods, one expects to uncover interesting interplays between hydrodynamics and diffusion. Here are some of the basic facts. When a low Reynolds number swimmer approaches another object it pushes it away. Objects tend to slide around each other without touching. Activities such as eating (or sex) become very tricky [27], [67].

Diffusion, on the other hand, brings small objects such as nutrients in contact with a microswimmer's surface according to the laws and time scales of Brownian motion. So, in this realm bacteria and protozoa live different realities. Microorganisms on the scale of $E$. coli and below are completely dependent on diffusion [4]. According to Berg, Purcell estimated in a "back-of-the-envelope" calculation that $1 \mu \mathrm{m}$ objects would have

\footnotetext{
${ }^{13}$ Mathematicians often have a solution looking for a problem.
} 
to expend enough energy to boil water in order to get much use out of using stirring to feed. However when the organism gets even $10 \mu \mathrm{m}$ bigger, stirring becomes a viable strategy (the essence of the calculation is that stirring efficiency is proportional to $R^{6}$ ).

Berg showed us a video, called "Death at Low Reynolds Number" (music from "Jaws") made for comic relief at conferences, in which rotifers and other animals hunt and eat $E$. coli. The predatory rotifers have an amazingly intricate structure of cilia which brings the $E$. coli into their gut. There is another predator which curls up into a small ball, and then unfurls itself like a frog tongue to suck up $E$. coli.

Berg also has amazing observations [6], [7] on the information processing and feeding strategies of $E$. coli. Their motions consist of a series of "runs"--nearly straight lines and "tumbles"-random changes of direction. Due to Brownian motion, E. coli are turned by ninety degrees about every 10 seconds. Any run that lasts longer than this is no longer close to straight. Because of this it does not make sense for E. coli to store information about its environment for any longer than 10 seconds. By subjecting $E$. coli to delta function-like pulses of nutrient concentrations and then observing their response, Berg was able to determine that they store information about chemical gradients for about 4 seconds. They integrate this information and it affects their swimming strategy. Essentially, if a chemical concentration is increasing in time, either due to their motion, or because of the biologist's pipette, then the runs become longer. Larger microorganisms may take advantage of a spatial process as well. Slime molds are able to steer toward nutrients. C. elegans, which are organisms composed of about 1000 cells and 300 neurons, follow nutritive gradients along spiral paths.

- Swimming enhanced diffusion. Can an animal's swimming stroke effect the rate of diffusive uptake of nutrients by an appreciable amount? Purcell's calculation suggests not. Berg and Purcell estimated the effect of swimming by using a diffusion equation with drift term provided by Stokes' flow past a sphere [4]. However, the flow field produced by a microorganism, seen from far away, is that of a Stokes' dipole, not that of flow past a sphere, which is a stokeslet (monopole). Nowadays, accurate description of the near-field of a bacterial motion is possible [72].

What is the effect of using this more accurate drift term? Are certain swimming strategies much better than others in terms of diffusive uptake? If so, has evolution taken advantage of them?

- Filter feeding. Small filter feeders clearly use Stokes' or near-Stokesian flow in order to amplify the diffusive uptake of nutrients. There are many strategies for doing this. A classic example of a filter feeder is the tunicate. They spend their life attached to the sea floor pumping sea water through their gut using a layer of densely packed cilia. Their hearts are reversible peristaltic pumps. See [28] for an analysis of these organisms from our point of view. One problem is to find a reasonable measure of efficiency for this strategy and to formulate the corresponding optimal design problems. The solutions can then be compared to what is found in nature.

- Collar flagellates. A mathematical theory was developed by Higdon [41].

- "Suckers". The ability of flagellar motion to produce pressure gradients for suction is exemplified in Giardia lamblia, a most popular intestinal parasite. Giardia uses its sucking disk for attachment into the mucosa [45]. 
- Calanoid copepods. These are planktonic crustaceans very important for the ecological food chain in oceans and lakes. The physiology of copepod feeding is quite intricate. High-speed movies have shown that copepods" appendages form a "dream team" of low Reynolds number basketball players-they are able to generate flows to direct particles of food into their gut [48]. It may be interesting to solve the idealized problem of controlling a particle using two rigid appendages. The Reynolds number range for the appendages of copepods, $10^{-2}$ to 1 , allows for different flow regimes, depending on the particular task at hand [49].

- Rotifers. Rotifers look like small vacuum cleaners as they draw E. coli into their gut. They generate currents around their oral cavity using a layer of densely packed cilia. It is possible that an envelope model can be used to describe the flows generated by the cilia surrounding the oral cavity of these organisms.

\subsection{Mystery Swimmers}

Interesting descriptions of microorganism locomotion can be found in [46], [70]. ${ }^{14} \mathrm{~A}$ mystery swimmer is a microorganism for which we have no explanation of how they swim. Find a reasonable explanation that is experimentally testable. Here are the two examples we know of.

- The Synechococcus. We learned of this swimmer from Berg. This is a bacterium living in sea water, with an important ecological role (carbon cycle). It is about $1 \mu \mathrm{m}$ in diameter, and swims fast, at $20 \mu \mathrm{m}$ per sec. It does not change shape, at least in a manner visible to the eye. It has no internal flagella. ${ }^{15}$ Motion by electrical means, and by "jets" have both been ruled out. So far, Berg was not able to glue tiny polystyrene balls on its surface. On a related organism, this was done, and one observes a strip of these balls streaming one way on or close to the organism's surface, and right next to them another strip of the balls streaming the opposite direction, as if they were on "conveyor belts." It seems unlikely that surface motion such as this could account for the swimming speeds of synechococcus as this would require it to transport its entire surface area down its length about 20 times a second. One mechanism which has not yet been ruled out is very small high-frequency oscillations of its surface. ${ }^{16}$ See the discussion in Section 3.

Remark. Synechococcus is related to certain "gliding organisms" [51]. These organisms latch onto the water surface and move along at a good clip $(2-3 \mu \mathrm{m} / \mathrm{sec})$; when they are separated from the surface they are unable to -swim, they only drift, being bounced around erratically by Brownian motion. Perhaps some gliding motions are done via

\footnotetext{
${ }^{14}$ Reynolds numbers seem to be ignored, as late as 1972, in [46].

15 Spirochetes were once mystery swimmers, until it was discovered that they have internal flagella. As they move inside the body, the external sheath rotates in opposite direction. In consequence, there is a translational velocity as well. It should be interesting to work out this calculation in detail. Here's an example where the tail wags the dog!

${ }^{16}$ While this paper was in the editorial process, $\mathrm{KE}, \mathrm{HB}$, and RM realized a very simple explanation that may solve the synechococcus mystery [29].
} 
classical nonholonomic mechanics. ${ }^{17}$ Motion of bacteria on solid surfaces or in modified fluids (e.g., suspensions of methyl cellulose and/or ficoll to obtain a "non-Newtonian" environment) requires them knowing other physical principles.

- "Rio's magnetotactic aggregate" [32]. A multicellular magnetotatic organism lives in the brackish water near the bottom of Rodrigues de Freitas Lagoon in Rio de Janeiro (a few blocks from Ipanema beach). This organism consists of about 20 cells; the overall size is about $4 \mu \mathrm{m}$. It swims at $30-100 \mu \mathrm{m} / \mathrm{sec}$, in quite a distinct manner. No cilia or flagellar bundles are observed. This aggregate could be a bacterial colony, but behaves as an individual organism. Our conjecture is that its motion may be a biological realization of "holonomy drive." The idealized model is a system of points connected by invisible rods, as discussed in the toy examples.

\subsection{Studies on Magnetotactic Bacteria}

General information on magnetotaxis can be found in [52]. Nogueira and Lins de Barros worked out an ab initio model for motion of bacteria (magnetotactic or not) [68]. Numerical solutions of the ODEs reproduce some finer details, which normally have been averaged out. For instance, the helical pattern around a "guiding-center" type averaged trajectory. We believe that their approach can benefit from some mathematical abstraction. In particular, can it be Hamiltonianized? For two or more bacteria, include interaction terms.

\subsection{Cooperative Behavior}

- Flagella of adjacent spermatozoa or flagellar bundles of adjacent bacteria tend to beat in unison, and they will go visibly faster. ${ }^{18}$ Is this a phase-locking phenomenon? Use Stokes' equations to explain the increase in speed.

- Coordination of bacterial flagella in a bundle seems to be mediated hydrodynamically, but the mechanism is not known. In ciliated protozoa, synchronization producing metachronal waves is most likely mechanically induced. Explain the mechanism. Again, could it be a phase-locking phenomenon? ${ }^{19}$

- Spirila is an elongated bacteria (about $50 \mu \mathrm{m}$ ) which swims back and forth. The flagellar bundles at the ends alternate clockwise (CW) and counterclockwise (CCW) rotation. The $\mathrm{CCW}$ bundle is in the direction of the body; the bundle undergoing $\mathrm{CW}$ motion is tilted. How is the switch coordinated?

- Bacterial flagella are rotated by independent motors [43]. What is the synchronization process by which a majority "vote" for CCW rotation?

\footnotetext{
${ }^{17}$ Imagine placing a small colony of these gliders on a wetted sphere, or pseudosphere. In this manner we could study low Reynolds number life in non-Euclidean geometries.

18 "Taylor calculated the forces acting to synchronize parallel undulating sheets and found these forces to be large for sheet spacings smaller than the wavelength of the undulations" [5].

${ }^{19}$ Cilia axonemes can be removed from the cells (using triton) and glued on glass. Exposed to ATP, metachronal waves are observed!
} 
- Some ciliated microorganisms have an isolated flagellum (Luis Henrique Leal, highspeed movies). The cilia perform an helicoidal wave, and the beating cycle of the flagellum is sharply in $1: 2$ resonance. Why?

\subsection{Patterns and Collective Phenomena}

- Traveling bands of bacteria. First seen just over hundred years ago by Engelmann and Pfeffer, such experiments can be done in science high-school classes. Traveling bands of magnetotactic bacteria, with different speeds, reminiscent to "solitons" have been observed in dense populations [78]. Organisms in a band travel side by side. A plausible hydrodynamical argument was given by D. C. Guell et al. [36] and we believe it can be elaborated in more detail.

- Spatio temporal patterns, self-organization. These have striking complexity, offering new opportunities for bifurcation theorists and experts on reaction-diffusion PDEs. Some references are [82], [21], [22]. We just mention some observations in [21]: for E. coli grown in a semi-solid agar culture, on a $7.5-\mathrm{cm}$ diameter dish, one observed first a sunflower-like array of spots. At smaller radii the spots tend to appear in circles, at larger radii on intersecting clockwise and counterclockwise spirals; other patterns are radial arrays of spots, spots and stripes, spots with radial tails arrayed in chevrons.

\subsection{Energy Expenditure}

- The bacterial motor. Berg has devoted much of his scientific life to the study of the structure and dynamics of the flagellar rotary motors of $E$. coli which he discovered in the early 1970s. We are not able to pose questions on this topic at this point, but we mention some references on this intriguing subject: [13], [8], [9], [10], [11], [12], [47], [60], [56], [61], [58].

- Microorganisms swim for fun and profit, having no energy consumption problems as far as locomotion is concerned. ${ }^{20}$ Their motor is powered directly by proton flux [13]. Efficiency is almost $100 \%$ at low speeds (in laboratory preparations), but drops to $1 \%$ at cruising speed. If energy consumption and efficiency issues arise, perhaps a Finsler metric should be chosen as the functional. If several organisms are involved one expects to obtain a variational problem of isoholonomic type involving the manybody connection and the grand resistance matrix. Is this biologically important? Can the problem be derived from first principles?

\subsection{Stochastic Processes in Bacterial Motion}

H. Berg developed the tracking microscope in the early 1970s, an instrument capable of following the motion of a single bacterium [14]. He found out that E. coli (and most other motile bacteria) move in a series of "runs" (lasting on average $1 \mathrm{sec}$ ) and "tumbles" (of about $0.1 \mathrm{sec}$ ). Runs are longer when the motion is in a positive gradient of a chemoattractant, but do not get shorter when in a negative direction (acuna-matata)!

${ }^{20}$ Purcell says it is like driving a Datsun in Saudi Arabia. 
- Consider a swarm of $E$. coli, sufficiently dense to interact hydrodynamically. It is plausible that the collective motion in the positive gradient will be enhanced, while the motions in other directions will be averaged out by the hydrodynamic interaction. How dense must the population be in order that this effect could be observed?

- Simultaneous optimization of two or more functions have been studied in Mathematical Economics. When there are two chemoattractants, can "Pareto-Smale sets" be observed?

- When particles are diffusing by Brownian motion, one gets the usual diffusion (heat) equation. Is it helpful to consider the low Reynolds hydrodynamical interaction? Inside a Stokes' flow, does it make sense to consider a subelliptic "grand-Laplacian"? (Its symbol would belong to a codimension- 6 distribution in phase space defined by zero total force and torque.)

We would like to end with another quote from E. O. Wilson's The Naturalist (p. 123):

I have evolved a rule that has proved useful for myself and might be for others not born with championship potential: for every level of mathematical ability there exists a field of science poorly enough developed to support original theory.

The advice I give to students in science is to move laterally and up and down and peer all around. If you have the will there is a discipline in which you can succeed. Look for the ones still thinly populated, ... be a hunter and explorer, not a problem solver...

\section{Acknowledgments}

We thank the participants of our seminar in Santa Cruz, especially students Andrew Klinger, Junko Hoshi, and Cesar Castilho; Professors T. Y. Wu and C. Brennen for their encouragement; and Howard Berg, for his beautiful talks and the many conversations in Rio. J. K. would like to thank Prof. Manuel de Leon for his hospitality at CSIC (Madrid, 1992), when some ideas started to take form. We also thank Darci Esquivel, Marcos Farina, and Henrique de Barros for providing an enthusiastic biophysical environment during Prof. Berg's visit. J. K. would like to acknowledge support provided by a Guggenheim Foundation fellowship, January-March 1994, to visit the Mathematics Department, University of California at Santa Cruz.

\section{References}

[1] Abraham, R. and Marsden, J. E.: Foundations of Mechanics, Addison-Wesley, Reading, MA (1978).

[2] Ambrose, W. and Singer, I. M.: A theorem on holonomy, Trans. AMS 75, 428-453 (1953).

[3] Arnold, V., Kozlov, V. V., and Neishtadt, A. I.: Dynamical Systems III, Encyclopaedia of Mathematical Sciences, Springer-Verlag, New York (1988).

[4] Berg, H.C.: Random Walks in Biology, expanded edition, Princeton University Press, Princeton, NJ (1993). 
[5] Berg, H. C. and Anderson, R. A.: Bacteria swim by rotating their flagellar filaments, Nature 245, 380-384 (1973).

[6] Berg, H. C. and Purcell, E. M.: Physics of chemoreception. Biophys. J. 20, 193-219 (1977).

[7] Berg, H. C.: Physics of bacterial chemotaxis, pp. 19-30 in Sensory Perception and Transduction in Aneural Organisms, eds. Colombetti, G., Lenci, F., and Song, P. S., Plenum, New York (1985).

[8] Berg, H.C.: Studies of motile bacteria. In Physics News, ed. by Schewe, P. F., American Institute of Physics, New York (1991).

[9] Berg, H. C.: Dynamic properties of bacterial flagellar motors. Nature 249, 77-79 (1974).

[10] Berg, H. C. and Khan, S.: A model for the flagellar rotary motor. In Mobility and Recognition in Cell Biology, pp. 485-497, eds. Sund, H. and Veeger, C., deGruyter, Berlin (1983).

[11] Berg, H. C., Manson, M. D., and Conley, M. P.: Dynamics and energetics of flagellar rotation in bacteria, Symp. Soc. Exp. Biol. 35, 1-31 (1982).

[12] Berg, H. C.: Dynamics and energetics of the bacterial rotary motor. In Protein Dynamics and Energy Transduction, pp. 312-344, ed. Ishiwata, S.-I., Taniguchi Foundation (1980).

[13] Berg, H.C.: Torque generation by the flagellar rotary motor, Biophys. J. 68, 163s-167s (1995).

[14] Berg, H. C.: How to track bacteria, Rev. Sci. Instrum. 42, 868-871 (1971).

[15] Blake, J. R.: Self propulsion due to oscillations on the surface of a cylinder at low Reynolds number, Bull. Austral. Math. Soc. 3, 255-264 (1971).

[16] Blake, J. R.: A spherical envelope approach to ciliary propulsion, J. Fluid Mech. 46, 199-208 (1971).

[17] Blake, J. R.: A model for the micro-structure in ciliated organisms, J. Fluid Mech. 55, 1-23 (1972).

[18] Brennen, C.: An oscillating boundary layer theory for ciliary propulsion, J. Fluid Mech. $\mathbf{6 5}$, 799-824 (1974).

[19] Brennen, C. and Winnet, H.: Fluid mechanics of propulsion by cilia and flagella, Ann. Rev. Fluid Mech. 9, 339-398 (1977).

[20] Brockett, R. W. and Dai, L: Non-holonomic kinematics and the role of elliptic functions in constructive controllability, in Nonholonomic Motion Planning, eds. Li, Z., Canny, J. F., Kluwer, Dordrecht (1993).

[21] Budrene, E. O. and Berg, H. C.: Complex patterns formed by motile cells of Escherichia coli, Nature 349, 630-633 (1991).

[22] Budrene, E. O. and Berg, H. C.: Dynamics of formation of symmetrical patterns of chemotactic bacteria, Nature 376, 49-53 (6 July 1995).

[23] Calleja, G. B.: Microbial Aggregation, CRC Press, Boca Raton, FL (1984).

[24] Childress, S.: Mechanics of Swimming and Flying, Cambridge University Press (1981).

[25] Chow, W. L.: Uber systeme van linearen partiellen differentialgleichungen ersten ordnung, Math. Ann. 117, 98-105 (1939).

[26] Chwang, A. T. and Wu, T. Y.: A note on the helical movement of micro-organisms, Proc. Roy. Soc. Lond. B. 178, 327-346 (1971).

[27] Curtis, H.: The Marvelous Animals: An Introduction to the Protozoa, The Natural History Press, New York (1968).

[28] Ehlers, K. M.: The Geometry of Swimming and Pumping at Low Reynolds Number, Ph.D. Thesis, University of California, Santa Cruz (1995).

[29] Ehlers, K. M, Berg, H. C., and Montgomery, R.: Do synechococcus swim using traveling surface waves?, Proc. Natl. Acad. Sci. USA 93, 8340-8344 (1996).

[30] England, H.: Complex Variable Methods in Elasticity, Wiley-Interscience, New York (1971).

[31] Fauci, L. J.: Computational modeling of the swimming of biflagellated algal cells, in Contemp. Math, 141, 91-102, ed. A. Y. Cheer, C. P. van Dam (1993).

[32] Farina, M., Esquivel, D. M. S., and Lins de Barros, H. G. P.: Magnetic iron-sulphur crystals from a magnetotactic microorganism, Nature 343, 6255, 256-258 (1990).

[33] Fearing, R. S.: Control of a micro-organism as a prototype micro-robot, Second Int. Symp. on Micromachines and Human Sciences, Nagoya, Japan, 1991.

[34] Flam, F.: Swarms of mini-robots set to take on Mars terrain, Science 257, 1621, (18 Sept. 1992). 
[35] Greenbaum, A., Greengard, L., and Mayo, A.: On the numerical solution of the biharmonic equation on the plane, Physica D 60(1-4), 216-225 (1992).

[36] Guell, D. C., Brenner, H., Frankel, R. B., and Hartman, H.: Hydrodynamic forces and band formation in swimming magnetotactic bacteria, J. Theor. Biol. 135, 525-542 (1988).

[37] Guichardet, A.: On rotation and vibration motions of molecules, Ann. Inst. H. Poincaré, Phys. Theor. 40(3), 329-342 (1984).

[38] Guillemin, V. and Sternberg, S.: Geometric Asymptotics, American Mathematical Society, Providence, RI (1977).

[39] Hasimoto, H. and Sano, H.: Stokeslets and eddies in creeping flow, Ann. Rev. Fluid Mech. 12, 335-364 (1980).

[40] Happel, J. and Brenner, H.: Low Reynolds Number Hydrodynamics, Kluwer Academic, Dordrecht (1991).

[41] Higdon, J. J. L.: The generation of feeding currents by flagellar motions, J. Fluid Mech. 94(2), 305-330 (1979).

[42] Hirsch, P.: Microcolony formation and consortia, in Microbial Adhesion and Aggregation, ed. K. C. Marshall, pp. 373-393, Springer-Verlag, New York (1984).

[43] Ishihara, A., Segall, J. E., Block, S. M., and Berg, H. C.: Coordination of flagella on filamentous cells of Escherichia coli, J. Bacteriol. 155, 228-237 (1983).

[44] Jahn, T. L., and Bovee, E. C.: Motile behavior of Protozoa, in Research in Protozoology, ed. Tze-Tuan Chen, vol. 1, Pergamon, New York (1967).

[45] Jones, R. D., Lemanski, C., and Jones, T. J.: Theory of attachment in Giardia, Biophys. J. 44, $185-190(1983)$.

[46] Jahn, T. L. and Votta, J. J.: Locomotion of protozoa, Ann. Rev. Fluid Mech. 4, 93-116 (1972).

[47] Khan, S., Meister, M., and Berg, H. C.: Constraints on flagellar rotation, J. Mol. Biol. 184, 645-656 (1985).

[48] Koehl, M. A. R.: Feeding at low Reynolds number by Copepods, Lect. Math. Life Sciences 14, 89-117 (1981).

[49] Koehl, M. A. R.: Hairy little legs: feeding, smelling and swimming at low Reynolds numbers, in Contemp. Math. 141, 33-64, Fluid dynamics in biology, eds. Cheer, A. Y., van Dam, C. P. (1993).

[50] Keller, S. T. and Wu, T. Y.: A porous prolate-spheroidal model for ciliated micro-organisms, J. Fluid Mech. 80(2), 259-278 (1977).

[51] Lapidus, R. and Berg, H. C.: Gliding motility of Cytophaga sp.Strain U67, J. Bacteriology 151(1), 384-398 (1982).

[52] Lins de Barros, H. G. P., Esquivel, D. M. S., and Farina, M.: Magnetotaxis, Sci. Progress Oxford 74, 347-359 (1990).

[53] Li, Z. and Canny, J. F. (eds.): Nonholonomic Motion Planning, Kluwer, Dordrecht (1993).

[54] Lighthill, J.: Biofluiddynamics: A survey, Contemp. Math. 141, 1-23 (1993), eds. Cheer, A. Y., van Dam, C. P.

[55] Lighthill, L.: Mathematical Biofluidmechanics, SIAM (1975).

[56] Lowe, G., Meister, M., and Berg, H. C.: Rapid rotation of flagellar bundles in swimming bacteria, Nature 325, 637-640 (1987).

[57] Ludwig, W.: Zur theorie der flimmerbewegung (dynamik, nutzeffekt, energiebilanz), Z. vergl. Physiol. 13, 397-504 (1930).

[58] Manson, M. D., Tedesco, P. M. and Berg, H. C.: Energetics of flagellar rotation in bacteria, J. Mol. Biol. 138, $541-561$ (1980).

[59] Marden, J.H. and Kramer, M. G.: Locomotor performance of insects with rudimentary wings, Nature 377, 332-334 (28 Sept. 1995).

[60] Meister, M., Lowe, G., and Berg, H.C.: The proton flux through the bacterial flagellar motor, Cell 49, 643-650 (1987).

[61] Meister, M. and Berg, H. C.: The stall torque of the bacterial flagellar motor, Biophys. J. 52, 413-419 (1987).

[62] Montgomery, R.: Gauge theory of the falling cat, Fields Institute Communications 1, 193-218 (1993). 
[63] Montgomery, R.: Isoholonomic problems and some applications, Commun. Math. Phys. 128, 565-592 (1990).

[64] Montgomery, R.: Nonholonomic control and gauge theory, in Nonholonomic Motion Planning, eds. Li, Z., Canny, J. F., Kluwer, Dordrecht (1993).

[65] Murray, R. M. and Sastry, S. S.: Steering nonholonomic control systems using sinusoids, in Nonholonomic Motion Planning, ed. Li, Z., Canny, J. F., Kluwer, Dordrecht (1993).

[66] Muskhelishvili, N. I.: Some Basic Problems of the Mathematical Theory of Elasticity, P. Noordhoff, Groningen. Holland (1953).

[67] Nisbet, B.: Nutrition and Feeding Strategies in Protozoa, Croom Helm, London and Camberra (1984).

[68] Nogueira, F. S. and Lins de Barros, H. G. P.: Study on the motion of magnetotactic bacteria, Eur. Biophys. J. 24, 13-21 (1995).

[69] Peskin, C. S., McQueen, D. M.: Computational biofluid dynamics, in Contemp. Math. 141, 161-186, ed. Cheer, A. Y., van Dam, C. P. (1993).

[70] Pedley, T. J. and Kessler, J. O.: Hydrodynamic phenomena in suspensions of swimming microorganisms, Ann. Rev. Fluid Mech. 24, 313-358 (1992).

[71] Purcell, E.: Life at low Reynolds number, Am. J. Phys. 45, 3-11 (1977).

[72] Ramia, M., Tullock, D. L., and Phan-Thien, N.: The role of hydrodynamic interaction in the locomotion of microorganisms, Biophys. J. 65, 755-778 (1993).

[73] Ramia, M. and Swan, M. A.: The swimming of unipolar cells of spirillum volutans: Theory and observations, J. Exp. Biol. 187, 75-100 (1994).

[74] Saier, M. H. and Jacobson, G. R.: The Molecular Basis of Sex and Differentiation, SpringerVerlag, New York (1984).

[75] Shapere, A. and Wilczek, F.: Geometry of self-propulsion at low Reynolds number, J. Fluid Mech. 198, 557-585 (1989).

[76] Shapere, A.: Gauge Theory of Deformable Bodies: A Theory of Something, Ph.D. thesis, Princeton University Physics Dept., Princeton, NJ (1989).

[77] Sizemore, D. R., Branstrom, A., and Sadoff, J. C.: Attenuatd Shigella as a DNA delivery vehicle for DNA-mediated immunization, Science 270, 299-302 (13 October 1995).

[78] Spormann, A. M.: Unusual swimming behavior of a magnetotactic bacterium, FEMS Microb. Ecol. 45, 37-45 (1987).

[79] Taylor, G. I.: Analysis of the swimming of microscopic organisms, Proc. Roy. Soc. Lond. A 209, 447-461 (1951).

[80] Taylor, G. I.: The action of waving cylindrical tails in propelling microscopic organisms, Proc. Roy. Soc. Lond. A 211, 225-239 (1952).

[81] Yates, G.: How microorganisms move through water, Am. Sci. 74, 358-365 (1986).

[82] Woodward, D. E., Tyson, R., Myerscough, M. R., Murray, J. D., Budrene, E. O., and Berg, H. C.: Spatio-temporal patterns generated by Salmonella typhimurium, Biophys. J. 682181 2189 (1995).

[83] Weinbaum, S. and Ganatos, P.: Numerical multipole and boundary integral equation techniques in Stokes flow, Ann. Rev. Fluid Mech. 22, 275-316, 1990.

[84] Wu, T. Y., Brennen, C., and Brokaw, C. (eds.): Swimming and Flying in Nature, vol, 1, Plenum, New York (1975). 